\title{
On self-similarity and stationary problem for fragmentation and coagulation models
}

\section{Solutions auto-similaires et stationnaires pour des modèles de fragmentation et de coagulation}

\author{
M. Escobedo ${ }^{a}$, S. Mischler ${ }^{b, *}$, M. Rodriguez Ricard ${ }^{c}$ \\ a Departamento de Matemáticas, Universidad del País Vasco, Apartado 644 Bilbao 48080, Spain \\ ${ }^{\mathrm{b}}$ Ceremade, Université Paris IX-Dauphine, place du M ${ }^{a l}$ DeLattre de Tasigny, Paris 75016, France \\ ${ }^{\mathrm{c}}$ Facultad de Matemática y Computación, Universidad de La Habana, C. Habana 10400, Cuba
}

Received 17 March 2004; accepted 11 June 2004

Available online 17 September 2004

\begin{abstract}
We prove the existence of a stationary solution of any given mass to the coagulation-fragmentation equation without assuming a detailed balance condition, but assuming instead that aggregation dominates fragmentation for small particles while fragmentation predominates for large particles. We also show the existence of a self-similar solution of any given mass to the coagulation equation and to the fragmentation equation for kernels satisfying a scaling property. These results are obtained, following the theory of Poincaré-Bendixson on dynamical systems, by applying the Tykonov fixed point theorem on the semigroup generated by the equation or by the associated equation written in "self-similar variables". Moreover, we show that the solutions to the fragmentation equation with initial data of a given mass behaves, as $t \rightarrow+\infty$, as the unique self similar solution of the same mass.

(C) 2005 L'Association Publications de l'Institut Henri Poincaré. Published by Elsevier B.V. All rights reserved
\end{abstract}

\section{Résumé}

Pour toute masse donnée, nous démontrons l'existence d'au moins une solution stationnaire pour l'équation de coagulationfragmentation. Nous ne faisons pas d'hypothèse d'équilibre en détails sur les coefficients mais nous supposons que la coagulation domine la fragmentation pour les particules de petite taille et que la fragmentation est prépondérante pour les particules de grande taille. Nous démontrons également l'existence de solutions auto-similaires pour l'équation de coagulation et pour l'équation de fragmentation sous une hypothèse d'homogéneité sur les noyaux. Ces résultats sont obtenus, s'inspirant de la preuve

\footnotetext{
* Corresponding author.

E-mail address: mischler@ceremade.dauphine.fr (S. Mischler).
} 
du Théorème de Poincaré-Bendixson, en appliquant le théorème de point fixe de Tykonov sur le semi-groupe engendré par l'équation ou par l'équation écrite en variables auto-similaires associée. Enfin, nous démontrons que les solutions de l'équation de fragmentation de masse donnée $\rho>0$ se comportent en temps grand comme la solutions auto-simialire de masse $\rho$.

(C) 2005 L'Association Publications de l'Institut Henri Poincaré. Published by Elsevier B.V. All rights reserved

MSC: 82C40; 60J75

Keywords: Equilibrium; No detailed balance condition; Poincaré-Bendixson's Theory; Tykonov fixed point theorem; Self-similar solutions;

Uniqueness; Existence; Convergence to self-similarity

\section{Introduction and notations}

We consider the Cauchy problem for a spatially homogeneous kinetic equation modelling the dynamic of a system of particles which undergo linear (secondary) fragmentation and/or aggregation events. More precisely, if we denote by $f(t, y) \geqslant 0$ the density of particles with mass $y \in \mathbb{R}_{+}:=(0, \infty)$ at time $t \geqslant 0$, we study the following equation

$$
\begin{aligned}
& \frac{\partial f}{\partial t}=Q(f) \quad \text { in } \mathbb{R}_{+} \times \mathbb{R}_{+}, \\
& f(0)=f_{\text {in }} \quad \text { in } \mathbb{R}_{+} .
\end{aligned}
$$

The coagulation-fragmentation operator $Q$ splits into two terms

$$
Q(f)=L f+C(f) .
$$

The first term, $L f$, describes the spontaneous fragmentation of one (mother) particle in several (possibly infinity) (daughters) particles. This process may be schematically written as

$$
\{y\} \rightarrow\left\{y^{(1)}\right\}+\cdots+\left\{y^{(k)}\right\}+\cdots
$$

with the mass conservation condition

$$
y^{(i)} \geqslant 0, \quad y=\sum_{k=1}^{\infty} y^{(k)}
$$

The linear fragmentation operator $L$ reads

$$
L f(y)=\int_{y}^{\infty} b\left(y^{\prime \prime}, y\right) f^{\prime \prime} d y^{\prime \prime}-f(y) \int_{0}^{y} \frac{y^{\prime}}{y} b\left(y, y^{\prime}\right) d y^{\prime},
$$

where $b=b\left(y, y^{\prime}\right)$ corresponds to the formation rate of particles of size $y^{\prime}$ by fragmentation of a particles of size $y^{\prime}$. Here and below, we use the shorthand notations $\psi=\psi(y), \psi^{\prime}=\psi\left(y^{\prime}\right)$ and $\psi^{\prime \prime}=\psi\left(y^{\prime \prime}\right)$ for any function $\psi$ on $\mathbb{R}_{+}$. We will consider a fragmentation rate $b$ satisfying

$$
b\left(y, y^{\prime}\right)=b_{0}(y) B\left(y^{\prime} / y\right),
$$

where $b_{0}$ is a function and $B$ is a measure such that

$$
b_{0}(y)=y^{\gamma}, \quad \gamma \geqslant-1, \quad B \geqslant 0, \quad \text { with } \operatorname{supp} B \subset[0,1], \quad \int_{0}^{1} y d B(y)<+\infty .
$$


The second term in the right-hand side of (1.3), $C(f)$, models the growth mechanism resulting from the encounter of two mother particles. This may be schematically written

$$
\{y\}+\left\{y^{\prime}\right\} \stackrel{a}{\longrightarrow}\left\{y^{\prime \prime}\right\}, \quad \text { with } y^{\prime \prime}=y+y^{\prime},
$$

where $a=a\left(y, y^{\prime}\right)$ is the rate of occurrence of the aggregation of two particles of mass $y$ and $y^{\prime}$. The operator $C$ is then given by:

$$
C(f)(y)=\frac{1}{2} \int_{0}^{y} a\left(y-y^{\prime}, y^{\prime}\right) f\left(y-y^{\prime}\right) f\left(y^{\prime}\right) d y^{\prime}-\int_{0}^{\infty} a\left(y, y^{\prime}\right) f(y) f\left(y^{\prime}\right) d y^{\prime} .
$$

We will consider a coagulation rate satisfying

$$
a\left(y, y^{\prime}\right)=y^{\alpha}\left(y^{\prime}\right)^{\beta}+y^{\beta}\left(y^{\prime}\right)^{\alpha}, \quad-1 \leqslant \alpha \leqslant 0 \leqslant \beta \leqslant 1, \lambda:=\alpha+\beta \in[0,1) .
$$

Remark 1.1. Hypothesis (1.8) is made in order to simplify the presentation. All the results presented in this paper are also true for a coagulation rate $a=a_{1}+\cdots+a_{I}$ where each of the functions $a_{i}, i \in 1, \ldots, I$, satisfy the assumption (1.8) and all the others hypothesis that are made in the paper (see (4.2), (4.3) and (5.2)). In particular, our results are true for the Smoluchowski coagulation rate

$$
a\left(y, y^{\prime}\right)=\left(y^{\nu}+\left(y^{\prime}\right)^{\nu}\right)^{\mu}\left(y^{\sigma}+\left(y^{\prime}\right)^{\sigma}\right),
$$

with $\nu=1 / 3, \mu=1$ and $\sigma=-1 / 3$.

The main motivation of this work is to describe the long time asymptotic behavior of the solutions to such type of coagulation and fragmentation equations. As it is well known, the stationary and self-similar solutions turn out to be important to describe such behavior. We are then led to consider first the existence of this type of particular solutions. These are two important questions both for the applications and from a mathematical point of view. Among the extensive physical and applied literature we may quote for example $[16,17,14,44,30,38]$ and references therein. In the mathematical literature, the study of self-similar solutions for the fragmentation equation has been studied using probabilistic methods in $[3,9,10]$, and the asymptotic behavior in $[6,7]$. The convergence to the equilibrium state for the coagulation-fragmentation equation with detailed balance condition is considered in [35] (see also the references therein), and without this condition in $[19,26]$. Finally, the self-similar solutions and asymptotic behavior for coagulation equation have been considered by probabilistic methods in [8,13], and in [39,40], for $a\left(y, y^{\prime}\right)=1$ and $a\left(y, y^{\prime}\right)=y+y^{\prime}$, using deterministic analytic methods. See also [12,21,31,32] for related works. (We do not pretend to be exhaustive at all.)

We do not consider here coagulation fragmentation equations whose solutions undergo finite time singularity formation such as gelation (loss of mass by the formation of "particles of infinite size" or gel) or shattering (loss of mass by the formation of "particles of zero size" or dust).

Therefore, in the present article, we only treat coagulation fragmentation equations which preserve the mass of the solutions for all time. This property is fundamental for all the results presented in this work. Under the conditions (1.6) and (1.8) the existence of global mass preserving solutions for coagulation fragmentation equations is classical, and well known. We refer to the recent bibliography as [33,22,25].

On the other hand, our purpose is not to prove the results under the greatest generality, nor to be exhaustive. We only want to get some insight into this problem using a simple argument. We then make simplifying hypothesis, such as (1.6) and (1.8) for the sake of clarity and brevity. Our strategy for proving existence result of particular solutions for such a coagulation and/or fragmentation equations follows the one presented in [28] in the Boltzmann equation context. It is based on the following simple abstract result, Theorem 1.2, on semigroups leaving invariant a convex and compact set, which is reminiscent yet in the theory of Poincaré-Bendixson on dynamical systems, see for instance [4] (Théorème 7.4). Theorem 1.2 is a simple consequence of the Tykonov fixed point theorem and it is a variant to similar results presented in $[4,2,28]$. 
Theorem 1.2. Let $Y$ be a Banach space and $\left(S_{t}\right)_{t} \geqslant 0$ be a continuous semigroup on $Y$. Assume that $S_{t}$ is weakly (sequentially) continuous for any $t>0$ and that there exists $\mathcal{Z}$ a nonempty convex and weakly (sequentially) compact subset of $Y$ which is invariant under the action of $S_{t}$ (that is $S_{t} z \in \mathcal{Z}$ for any $z \in \mathcal{Z}$ and $t \geqslant 0$ ). Then, there exists $z_{0} \in \mathcal{Z}$ which is stationary under the action of $S_{t}$ (that is $S_{t} z_{0}=z_{0}$ for any $t \geqslant 0$ ).

Proof of Theorem 1.2. For any $t>0$, thanks to the Tykonov point fixed theorem (see for instance [20, pp. 161163]), there exists $z_{t} \in \mathcal{Z}$ such that $S_{t} z_{t}=z_{t}$. On the one hand,

$$
S_{i 2^{-m}} z_{2^{-n}}=z_{2^{-n}} \quad \text { for any } i, n, m \in \mathbb{N}, m \leqslant n .
$$

On the other hand, by weak compactness of $\mathcal{Z}$, we may extract a subsequence $\left(z_{2}-n_{k}\right)_{k}$ which converges weakly to a limit $z_{0} \in \mathcal{Z}$. By weak continuity of $S_{t}$ we may pass to the limit $n_{k} \rightarrow \infty$ in (1.9) and we obtain $S_{t} z_{0}=z_{0}$ for any dyadic time $t \geqslant 0$. We conclude that $z_{0}$ is stationary by continuity of $t \mapsto S_{t}$ and density of the dyadic real numbers in the real line.

The proof of the existence of steady solutions using this abstract result is a slight modification of the method used in [28] for granular flows equations. It has also been used in [5] to prove existence of stationary solutions for the Boltzmann-Pauli equation and in [41] to prove existence of self-similar profiles for the inelastic Boltzmann equation of granular flows.

Once the existence of stationary or self-similar solutions is established, one may conjecture that these particular solutions should describe the long time behavior of the solutions of the Cauchy problem (1.1), (1.2) for a certain set of initial data $f_{\text {in }}$. Nevertheless, we are only able to prove this result for the fragmentation equation, i.e. when $a \equiv 0$. This is done using, very classically, the uniqueness of the asymptotic state and the existence of a strict Lyapunov functional. These two facts are not known for the general coagulation fragmentation equation or for the self-similar profile equation associated to the coagulation equation. For all these questions and more, the interested reader may consult the surveys $[1,18,36,38]$.

We describe now rather briefly the contents of this paper. Let us start saying that, when the two terms in Eq. (1.3) are present, we look for a stationary solution. We search then, for any given mass $\rho>0$, a solution $F_{\rho}$ to the stationary problem

$$
Q_{\mathrm{CF}}(f):=C(f)+L f=0 \quad \text { and } \quad M_{1}(f)=\rho,
$$

where $M_{1}(f)=\int_{0}^{\infty} y f d y$ is the mass.

But, if only one of the two terms $C(f)$ or $L f$ is present, the only stationary solution is the trivial one $f \equiv 0$ and we look then for mass preserving self-similar solutions. These solutions are of the form

$$
f(t, y)=t^{2 v} g\left(t^{v} y\right)
$$

for some exponent $v=(1+\gamma)^{-1}>0$ for the fragmentation equation and $v=-(1-\lambda)^{-1}<0$ for the coagulation equation. The function $g$, called sometimes the self-similar profile, satisfies a stationary equation of the form:

$$
D g-(1+\gamma) L g=0 \quad \text { and } \quad M_{1}(g)=\rho,
$$

for the fragmentation equation, and

$$
D g+(1-\lambda) C(g)=0 \quad \text { and } \quad M_{1}(g)=\rho,
$$

for the coagulation equation, where $D$ is the following linear transport operator preserving mass

$$
D g=2 g+y g_{y} .
$$

It is then natural to consider the following family of equation

$$
\partial_{t} g=\widetilde{Q}(g)
$$


where,

$$
\widetilde{Q}(g)=\varepsilon_{0} D(g)+\varepsilon_{1} L g+\varepsilon_{2} C(g),
$$

with $\varepsilon_{0} \in\{-1,0,1\}, \varepsilon_{1}, \varepsilon_{2} \in\{0,1\}$. There exists a strong and explicit relation between the solutions $f$ of Eq. (1.2) where only the coagulation or the fragmentation are present and the solutions $g$ of (1.14) with $\varepsilon_{1} \varepsilon_{2}=0$. Suppose for example that $g$ is a solution to (1.14) with $\varepsilon_{0}=-1, \varepsilon_{1}=1+\gamma 0$ and $\varepsilon_{2}=0$. Then, the function

$$
f(t, y)=(1+t)^{\frac{2}{1+\gamma}} g\left(\frac{1}{1+\gamma} \ln (1+t),(1+t)^{\frac{1}{1+\gamma}} y\right)
$$

satisfies the fragmentation equation (1.1) (with $C(f) \equiv 0$ ). Similarly, if $g$ is a solution to (1.14) with $\varepsilon_{1}=0$ and $\varepsilon_{0}=1$. Then, the function

$$
f(t, y)=(1+t)^{-\frac{2}{1-\lambda}} g\left(\frac{1}{1-\lambda} \ln (1+t),(1+t)^{-\frac{1}{1-\lambda}} y\right)
$$

satisfies the coagulation equation (1.1) (with $L f \equiv 0$ ).

On the other hand, if $f$ satisfies the fragmentation equation, we obtain a solution $g$ to (1.14) with $\varepsilon_{0}=-1$, $\varepsilon_{1}=\gamma+1$ and $\varepsilon_{2}=0$, defining

$$
g(t, y)=e^{-2 t} f\left(e^{(1+\gamma) t}-1, y e^{-t}\right),
$$

and if $f$ satisfies the coagulation equation, we obtain a solution $g$ to (1.14) with $\varepsilon_{0}=1, \varepsilon_{1}=0$ and $\varepsilon_{2}=1-\lambda$, defining

$$
g(t, y)=e^{2 t} f\left(e^{(1-\lambda) t}-1, y e^{t}\right) .
$$

This well known property of the self-similar change of variables has already been extensively used for the study of the long time behavior of the solutions to partial differential equations (see for example [24] and the references therein) and is also used here. In particular, all our results about the existence or the uniqueness of solutions to Eq. (1.14) will automatically give an existence or uniqueness result for the corresponding coagulation or fragmentation equation.

The rest of the paper is organized as follows. In Section 2 we define precisely the notions of solutions used in all this work. We prove uniqueness and stability of solutions to the growth equation (1.14), (1.15), and existence for "truncated" rate functions $a$ and $b$. These results will be very useful in the following sections.

In Section 3 we consider the fragmentation equation. We show the existence, for any given mass $\rho>0$, of a unique self-similar solution. Then we prove that this solution describes the long time behavior of the solutions to the Cauchy problem with initial data $f_{\text {in }}$ of mass $\rho$.

In Section 4 we consider the coagulation fragmentation equation without detailed balance condition. We prove the existence, for any given mass $\rho>0$, of a stationary solution. Finally, we treat in Section 5 the coagulation equation and prove the existence, for any given mass $\rho>0$, of a unique self-similar solution.

\section{Generalities and tool box}

We gather in this section some elementary results about the Cauchy problem associated to the growth equation (1.14), (1.15). Since all of them are very classical the proofs will only be sketched. For further developments and more precise statements and proofs we refer, for instance, to $[36,33,25]$ and the numerous references therein.

We first present some notations. We denote by $L_{\text {loc }}^{1}$ the space of integrable functions $f:(0, \infty) \rightarrow \mathbb{R}$ on any compact $[\varepsilon, 1 / \varepsilon], \varepsilon \in(0,1)$ and by $M_{\text {loc }}^{1}$ the associated measures spaces. For any given continuous function $\varphi:(0, \infty) \rightarrow(0, \infty)$, we define

$$
M_{\varphi}^{1}:=\left\{\mu \in M_{\mathrm{loc}}^{1} \text {, such that } M_{\varphi}(|\mu|)<\infty\right\}, \quad L_{\varphi}^{1}:=M_{\varphi}^{1} \cap L_{\mathrm{loc}}^{1},
$$


where for any measure $0 \leqslant v \in M_{\text {loc }}^{1}$, we define the generalized moment $M_{\varphi}(v)$ by

$$
M_{\varphi}(v):=\int_{0}^{\infty} \varphi(y) d v(y) .
$$

In order to shorten notations we also (abusively) denote, for any $k \in \mathbb{R}$,

$$
\dot{M}_{k}^{1}=M_{y^{k}}^{1}, \quad \dot{L}_{k}^{1}=L_{y^{k}}^{1}, \quad M_{k}^{1}=M_{1+y^{k}}^{1}, \quad L_{k}^{1}=L_{1+y^{k}}^{1} .
$$

Finally, we define

$$
\dot{B} V_{1}:=\left\{f \in L_{\text {loc }}^{1} \text {, such that } f^{\prime} \in \dot{M}_{1}^{1}\right\} .
$$

The same construction is made on the subset $(0,1)$. In that case, $L_{\text {loc }}^{1}(0,1)$ is the set of measurable functions, integrable on $[\varepsilon, 1]$ for any $\varepsilon>0$. Theses spaces are always denoted indicating the interval $(0,1)$, like for instance $\dot{M}_{k}^{1}(0,1), \dot{L}_{k}^{1}(0,1), M_{k}^{1}(0,1), L_{k}^{1}(0,1), \dot{B} V_{1}(0,1)$. Let us emphasize that all these are Banach spaces.

We show in the next lemma how to define the different growth operators $L, C$ and $D$ in the strong and weak sense that will be needed later.

Lemma 2.1. For any $k \in \mathbb{R}$,

$$
f \in \dot{L}_{\alpha+\min (0, k)}^{1} \cap \dot{L}_{\beta+\max (0, k)}^{1} \Rightarrow C(f) \in L_{k}^{1},
$$

and, if $B \in \dot{L}_{1}^{1} \cap \dot{L}_{k}^{1}$,

$$
f \in \dot{L}_{\gamma+1}^{1} \cap \dot{L}_{\gamma+1+k}^{1} \Rightarrow L f \in \dot{L}_{k}^{1} .
$$

For any $\mu \in \dot{M}_{\alpha+1}^{1} \cap \dot{M}_{\beta+1}^{1}$ and $\phi \in C_{c}^{1}(0, \infty)$, the duality product

$$
\langle C(\mu), \phi\rangle:=\frac{1}{2} \int_{0}^{\infty} \int_{0}^{\infty} a\left(\phi^{\prime \prime}-\phi-\phi^{\prime}\right) d \mu(y) d \mu\left(y^{\prime}\right)
$$

is well defined.

For any $\mu \in \dot{M}_{\gamma+1}^{1}+\dot{M}_{\gamma+2}^{1}$ and $\phi \in C_{c}^{1}(0, \infty)$, the duality product

$$
\langle L \mu, \phi\rangle:=\left\langle\mu, L^{*} \phi\right\rangle, \quad\left(L^{*} \phi\right)(y)=\int_{0}^{y} b\left(y, y^{\prime}\right)\left[\phi^{\prime}-\frac{y^{\prime}}{y} \phi\right] d y^{\prime}
$$

is well defined.

For any $\mu \in M_{\mathrm{loc}}^{1}(0, \infty)$ and $\phi \in C_{c}^{1}(0, \infty)$ the duality product

$$
\langle D \mu, \phi\rangle:=\left\langle\mu, D^{*} \phi\right\rangle, \quad D^{*}(\phi)=2 \phi-(y \phi)_{y}
$$

is well defined.

Proof of Lemma 2.1. For any $\phi \in C_{c}^{2}(0, \infty)$, we write $\phi=y \zeta(y)$ with $\zeta \in C_{c}^{1}(0, \infty)$. Then

$$
\phi^{\prime \prime}-\phi-\phi^{\prime}=y\left(\zeta^{\prime \prime}-\zeta\right)+y^{\prime}\left(\zeta^{\prime \prime}-\zeta^{\prime}\right)=y y^{\prime} \psi\left(y, y^{\prime}\right)
$$

with $\psi \in C_{b}\left((0, \infty)^{2}\right)$, since $\zeta^{\prime \prime}-\zeta=y^{\prime} \frac{d \zeta}{d y}\left((1-s) y+s y^{\prime}\right)=: y^{\prime} \psi_{1}\left(y, y^{\prime}\right)$. Therefore,

$$
\langle C(f), \phi\rangle=\frac{1}{2} \int_{0}^{\infty} \int_{0}^{\infty} a y y^{\prime} \psi f f^{\prime} d y d y^{\prime},
$$

and $a y y^{\prime}=y^{1+\alpha}\left(y^{\prime}\right)^{1+\beta}+\left(y^{\prime}\right)^{1+\alpha} y^{1+\beta} \leqslant\left(y^{1+\alpha}+y^{1+\beta}\right)\left(\left(y^{\prime}\right)^{1+\alpha}+\left(y^{\prime}\right)^{1+\beta}\right)$. 
For any $\phi \in C_{c}^{1}(0, \infty)$, we write $\phi=y \zeta(y)$ with $\zeta \in C_{c}(0, \infty)$. Then

$$
\left|\left(L^{*} \phi\right)(y)\right|=\left|\int_{0}^{y} y^{\gamma} B\left(y^{\prime} / y\right) y^{\prime}\left[\zeta^{\prime}-\zeta\right] d y^{\prime}\right| \leqslant 2 y^{\gamma+2}\|B\|_{\dot{L}_{1}^{1}}\|\zeta\|_{L^{\infty}}
$$

and

$$
\left|\left(L^{*} \phi\right)(y)\right| \leqslant 2 y^{\gamma+1}\|B\|_{L_{1}^{1}}\|\phi\|_{L^{\infty}} .
$$

In the next lemma, whose proof is straightforward and is skipped, we associate a semigroup $S_{D}$ to the operator $D$.

Lemma 2.2. For any $h_{\mathrm{in}} \in \dot{L}_{k}^{1}$ there exists a unique solution $h \in C\left([0, T] ; \dot{L}_{k}^{1}\right)$ to the equation

$$
\partial_{t} h=D h, \quad h(0, .)=h_{\text {in }},
$$

which is given by

$$
h(t, y)=\left(S_{D} h_{\text {in }}\right)(t, y):=e^{2 t} h_{\text {in }}\left(e^{t} y\right) .
$$

As a consequence, for any $H \in L^{1}\left((0, T) ; \dot{L}_{k}^{1}(0, \infty)\right)$ and any $h_{\mathrm{in}} \in \dot{L}_{k}^{1}$, the unique solution $h \in C\left([0, T] ; \dot{L}_{k}^{1}\right)$ to the equation

$$
\partial_{t} h=2 h+y \partial_{y} h+H, \quad h(0, .)=h_{\text {in }},
$$

is given by the Duhamel formula

$$
h(t, y)=e^{2 t} f_{\text {in }}\left(e^{t} y\right)+\int_{0}^{t} e^{2(t-s)} H\left(s, e^{t-s} y\right) d s .
$$

We define now the notions of dual and mild solutions, as two kinds of weak solutions, that are used in the rest of this paper. First, we define the natural space of weak (dual) solution with finite mass by $X^{\text {dual }}=X_{c}$ for the coagulation equation, $X^{\text {dual }}=X_{f}$ for the fragmentation equation and $X^{\text {dual }}=X_{\mathrm{cf}}$ for the coagulation-fragmentation equation, where

$$
X_{c}:=\dot{M}_{\alpha+1}^{1} \cap \dot{M}_{\beta+1}^{1}, \quad X_{f}:=\dot{M}_{\max (1, \gamma+1)}^{1} \cap \dot{M}_{1}^{1}, \quad X_{\mathrm{cf}}:=\dot{M}_{\alpha+1}^{1} \cap \dot{M}_{\max (\beta, \gamma)+1}^{1} .
$$

Definition 2.3. Let be $\mu^{\text {in }} \in X^{\text {dual }}$, with $X^{\text {dual }}$ defined depending of the values of $\varepsilon_{i}$. A weak solution to (1.14) with initial datum $\mu^{\text {in }}$ is a nonnegative measure $\mu \in L^{\infty}\left(0, T ; X^{\text {dual }}\right)$ which satisfies $M_{1}(t)=M_{1}(0)$ for $t \geqslant 0$ and the duality equation

$$
\int_{0}^{\infty} \int_{0}^{\infty} \mu \partial_{t} \psi d y d t+\int_{0}^{\infty} \mu^{\mathrm{in}} \psi(0, .) d y+\int_{0}^{\infty}\langle\widetilde{Q}(\mu), \psi\rangle d t=0
$$

for each $\psi \in \mathcal{C}_{0}^{\infty}([0,+\infty) \times(0, \infty))$, where

$$
\langle\widetilde{Q}(\mu), \psi\rangle:=\varepsilon_{0}\langle D \mu, \psi\rangle+\varepsilon_{1}\langle L \mu, \psi\rangle+\varepsilon_{2}\langle C(\mu), \psi\rangle
$$

and the three terms at the right-hand side are defined thanks to the dual formulation presented in Lemma 2.1.

We also define the natural space of weak (mild) solutions with finite mass by $Y^{\text {mild }}=Y_{\mathrm{c}}, Y^{\text {mild }}=Y_{\mathrm{f}}$ or $Y^{\text {mild }}=$ $Y_{\mathrm{cf}}$, where now

$$
Y_{\mathrm{c}}:=\dot{L}_{\alpha}^{1} \cap \dot{L}_{1}^{1}, \quad Y_{\mathrm{f}}:=\dot{L}_{\gamma+1}^{1} \cap \dot{L}_{1}^{1}, \quad Y_{\mathrm{cf}}:=\dot{L}_{\alpha}^{1} \cap \dot{L}_{\max (\gamma+1,1)}^{1} .
$$


Definition 2.4. Let be $f^{\text {in }} \in Y^{\text {mild }}$. A mild solution to (1.14) with initial datum $f^{\text {in }}$ is a nonnegative function $f \in C\left([0, T] ; \dot{L}_{1}^{1}\right) \cap L^{\infty}\left(0, T ; Y^{\text {mild }}\right)$ which satisfies $M_{1}(t)=M_{1}(0)$ for $t \geqslant 0$ and one of the three (equivalent) formulation of Eq. (1.14):

1. The mild sense

$$
f=S_{0}(t) f_{\text {in }}+\int_{0}^{t} S_{0}(t-s)\left(\varepsilon_{1} L f(s)+\varepsilon_{2} C(f)(s)\right) d s,
$$

where $S_{0}$ is the semigroup associated to $\varepsilon_{0} D$;

2. The distributional sense

$$
\int_{0}^{T} \int_{0}^{\infty}\left\{f \frac{\partial \phi}{\partial t}+\left(\varepsilon_{1} L f+\varepsilon_{2} C(f)\right) \phi-\varepsilon_{0} f D^{*} \phi\right\} d y d t=\int_{Y} f_{\text {in }} \phi(0, .) d y,
$$

for any $t>0$ and any $\phi \in C_{c}^{1}([0, T) \times(0, \infty))$;

3. The renormalized sense (which nothing but the chain rule)

$$
\frac{d}{d t} \int_{Y} \beta(f) \phi d y=\int_{0}^{\infty}\left\{\left(\varepsilon_{1} L f+\varepsilon_{2} C(f)\right) \beta^{\prime}(f) \phi-\varepsilon_{0} f D^{*} \phi\right\} d y,
$$

in $\mathcal{D}^{\prime}([0, T))$, for any $\beta \in C^{1}(\mathbb{R}) \cap W^{1, \infty}(\mathbb{R}), \phi \in L^{\infty}(0, \infty)$, and $f(0,)=.f_{\text {in }}$.

Remark 2.5. We refer to $[15,33]$ for the proof of the equivalence of these three formulations. Since the semigroup $S_{D}$ is well defined on $\dot{M}_{k}^{1}$ one can also define mild solutions with $Y^{\text {mild }}$ given by $M_{\alpha+1}^{1} \cap \dot{M}_{\beta+1}^{1}$, $M_{\max (1, \gamma+1)}^{1} \cap \dot{M}_{1}^{1}$, or $\dot{M}_{\alpha+1}^{1} \cap \dot{M}_{\max (\beta, \gamma)+1}^{1}$.

The following result is a straightforward consequence of Lemma 2.1 and of the two definitions above.

Lemma 2.6. Assume $f \in L^{\infty}\left([0, T] ; X^{\text {dual }} \cap Y^{\text {mild }}\right)$. Then $f$ is a dual solution of (1.14) if, and only if, it is a mild solution of (1.14).

From now on, we will only call weak solution of (1.14) a dual solution or a mild solution. We present now a useful stability principle for weak solutions.

Theorem 2.7. Let $X$ be a Banach space such that the inclusion $X^{\prime} \subset X$ is weakly compact. Consider a sequence $\mu^{n}$ of weak solutions to the growth equation (1.14) and assume that $\left(\mu^{n}\right)$ is bounded in $L^{\infty}\left(0, T ; X^{\prime}\right)$. Then, there exists a nonnegative measure $\mu \in L^{\infty}(0, T ; X)$ and a subsequence $\left(\mu^{n_{k}}\right)$ such that $\mu^{n_{k}} \rightarrow \mu$ in the sense of distribution $\mathcal{D}^{\prime}((0, T) \times(0, \infty))$. Moreover $\mu$ is still a weak solution to the growth equation (1.14).

Proof of Theorem 2.7. It is a straightforward consequence of the weak compact injection $X^{\prime} \subset X$ and of the weak formulation given in Lemma 2.1, in which it is easy to pass to the limit. We only point out that, since $\mu^{n}$ satisfies Eq. (2.5) or (2.6), the sequence

$$
\left(\int_{0}^{\infty} \phi(y) \mu^{n}(t, d y)\right)
$$

is strongly compact in $C([0, T])$ for any $\phi \in \mathcal{D}(0, \infty)$, and any $T>0$. It is then possible to pass to the limit in the (quadratic) coagulation term. 
A natural and classical strategy to prove the existence of solutions to (1.14) is to obtain an existence result for some truncated or regularised rate functions, then to obtain a priori estimates, strongly dependent on each particular model and of the hypothesis on the rates functions, and to use finally the stability result Theorem 2.7.

Our next lemma concerns the growth equation (1.14) with "truncated" rate functions $a$ and $b$. It will be extended to more general functions $a$ and $b$ in each of the three different cases $\left(\varepsilon_{0}=0, \varepsilon_{1}=0\right.$ or $\left.\varepsilon_{2}=0\right)$ that are considered in the following sections.

Lemma 2.8. Consider the growth equation (1.14) and assume that the coagulation and fragmentation rates satisfy

$$
0 \leqslant a \in L^{\infty}\left(\mathbb{R}_{+}^{2}\right), \quad 0 \leqslant b_{0}, B \in L^{\infty}\left(\mathbb{R}_{+}\right)
$$

and

$$
\operatorname{supp} B \subset(0,1], \quad \operatorname{supp} b_{0} \text { is a compact of } \mathbb{R}_{+} .
$$

Then, for any $0 \leqslant f_{\text {in }} \in \dot{L}_{1}^{1}$, there exists a unique global weak solution $0 \leqslant f \in C\left([0, \infty) ; \dot{L}^{1}\right)$ to the growth equation (1.14). Moreover, if $f_{\text {in }} \in \dot{L}_{k}^{1}$ for some $k \in \mathbb{R}$, then $f \in L^{\infty}\left(0, T ; \dot{L}_{k}^{1}\right)$ for any $T>0$; if $f_{\text {in }} \in L^{p}$ for some $p>1$ then $f \in L^{\infty}\left(0, T ; L^{p}\right)$ for any $T>0$.

Proof of Lemma 2.8. For any $T, \lambda>0$ and $h \in C\left([0, T] \dot{L}_{1}^{1}\right)$ we define

$$
(\Phi h)(t):=S_{\lambda}(t) f_{\text {in }}+\int_{0}^{t} S_{\lambda}(t-s)(C(h)(s)+L h(s)) d s,
$$

where $S_{\lambda}$ is the semigroup associated to the equation $\partial_{t} g+\lambda g=\varepsilon_{0} D g$. We easily see that for $\lambda$ large enough the map $\Phi$ preserves the positivity and that for $T$ small enough the map $\Phi$ is a contraction in $C\left([0, T] \dot{L}_{1}^{1}\right)$. Therefore by the Banach fixed point theorem there exists a unique $f \in C\left([0, T] ; \dot{L}^{1}\right)$ such that $\Phi f=f$, and this is precisely a mild solution to (1.14). By an iterative argument we may choose $T$ arbitrary large. Finally, we may establish the a priori bounds

$$
\sup _{[0, T]}\|f\|_{\Xi} \leqslant C_{T}\left\|f_{\text {in }}\right\|_{\Xi} \quad \text { for } \Xi=\dot{L}_{k}^{1}, \quad \Xi=L^{p}
$$

and this is enough to conclude the proof. We refer for instance to [34] for more details.

We finally present a general uniqueness result for the growth equation (1.14).

Theorem 2.9. Assume that the rate functions $a$ and $b$ satisfy (1.5), (1.6), (1.8) and consider the growth equation (1.14), (1.15). When $\varepsilon_{2}=0$, there exists at most one (mild) solution in $\mathcal{C}\left([0, T] ; \dot{L}_{k}^{1}\right)$ for all $T>0$, for any $k \geqslant 1$. When $\varepsilon_{2} \neq 0$, if $B \in \dot{M}_{2 \alpha}^{1}$ there exists at most one (mild) solution in $\mathcal{C}\left([0, T] ; \dot{L}_{\alpha}^{1} \cap \dot{L}_{k}^{1}\right) \cap L^{1}\left(0, T ; \dot{L}_{2 \alpha}^{1} \cap\right.$ $\left.\dot{L}_{\beta+k}^{1}\right)$ with $k:=\max (\beta, \gamma+1+\alpha)$ if $\varepsilon_{1}>0$ and $k:=\beta$ if $\varepsilon_{1}=0$.

Proof of Theorem 2.9. Consider two solutions $f$ and $g$ to (1.14), (1.15) which belong to the functional space as stated above. Let introduce the function $\varphi=\varphi_{1}+\varphi_{2}$ with $\varphi_{i}=c_{i} y^{k_{i}}, c_{i} \geqslant 0, k_{i} \in \mathbb{R}, k_{1}<k_{2}$ and $k_{1} \leqslant 1$. We multiply the equation satisfied by $f-g$ by $\psi=\operatorname{sign}(f-g) \varphi$ and we integrate in the $y$ variable

$$
\begin{aligned}
\frac{d}{d t} \int_{0}^{\infty}|f-g| \varphi d y \leqslant & \varepsilon_{0} \int_{0}^{\infty}(f-g)\left(2 \psi-(y \psi)_{y}\right) d y+\frac{\varepsilon_{2}}{2} \int_{0}^{\infty} \int_{0}^{\infty} a(f-g)\left(f^{\prime}+g^{\prime}\right)\left(\psi^{\prime \prime}-\psi-\psi^{\prime}\right) d y d y^{\prime} \\
& +\varepsilon_{1} \int_{0}^{\infty}(f-g)(y) \int_{0}^{y} b\left(y, y^{\prime}\right)\left(\psi\left(y^{\prime}\right)-\frac{y^{\prime}}{y} \psi(y)\right) d y^{\prime} d y .
\end{aligned}
$$


On the one hand, the subadditivity of $\varphi_{1}$ (that is $\varphi_{1}^{\prime \prime} \leqslant \varphi_{1}+\varphi_{1}^{\prime}$ ) ensures that

$$
\begin{aligned}
a(f-g)\left(\psi_{1}^{\prime \prime}-\psi_{1}-\psi_{1}^{\prime}\right) & \leqslant a|f-g|\left(\varphi_{1}^{\prime \prime}-\varphi_{1}+\varphi_{1}^{\prime}\right) \\
& \leqslant 2|f-g|\left(y^{\alpha}\left(y^{\prime}\right)^{k_{1}+\beta}+y^{\beta}\left(y^{\prime}\right)^{k_{1}+\alpha}\right) .
\end{aligned}
$$

The same holds with $\varphi_{2}$ if $k_{2} \leqslant 1$, and when $k_{2}>1$ we may use the elementary inequality

$$
\left(y+y^{\prime}\right)^{k_{2}}-y^{k_{2}}+\left(y^{\prime}\right)^{k_{2}} \leqslant C_{k_{2}}\left(y^{k_{2}-1} y^{\prime}+\left(y^{\prime}\right)^{k_{2}}\right)
$$

to obtain

$$
a(f-g)\left(\psi_{2}^{\prime \prime}-\psi_{2}-\psi_{2}^{\prime}\right) \leqslant C_{k_{2}}^{\prime}|f-g|\left(y^{\alpha}+y^{\beta+k_{2}-1}\right)\left(\left(y^{\prime}\right)^{\alpha+1}+\left(y^{\prime}\right)^{\beta+k_{2}}\right) .
$$

In any case, taking $\varphi=c_{1} y^{\alpha}+c_{2} y^{k}$, with $k$ defined in the statement of the theorem, we obtain

$$
\langle C(f)-C(g), \psi\rangle \leqslant K \int_{0}^{\infty}|f-g| \varphi d y \int_{0}^{\infty}\left(f^{\prime}+g^{\prime}\right)\left(\left(y^{\prime}\right)^{2 \alpha}+\left(y^{\prime}\right)^{k+\beta}\right) d y .
$$

On the other hand, we have

$$
(f-g) L^{*} \psi_{2} \leqslant|f-g| L^{*} \varphi_{2} \leqslant 0
$$

and

$$
(f-g) L^{*} \psi_{1} \leqslant|f-g| L^{*} \varphi_{1} \leqslant|(f-g)(y)|\left(L^{*} y^{k}\right)=\|B\|_{\dot{M}_{k_{1}}^{1} \cap \dot{L}_{1}^{1}}(1-k) y^{\gamma+1+k} .
$$

Finally, we notice that $\left|2 \varphi-(y \varphi)_{y}\right| \leqslant K^{\prime} \varphi$ for some constant $K^{\prime}=K^{\prime}(\varphi)$, and then

$$
\left|(f-g)\left(2 \psi-(y \psi)_{y}\right)\right|=|| f-g\left|\left(2 \varphi-(y \varphi)_{y}\right)\right| \leqslant K^{\prime} \varphi|f-g| .
$$

Therefore,

$$
\frac{d}{d t} \int_{Y}|f-g| \varphi d y \leqslant K \int_{Y}|f-g| \varphi d y \int_{0}^{\infty}\left(f^{\prime}+g^{\prime}\right)\left(\left(y^{\prime}\right)^{2 \alpha}+\left(y^{\prime}\right)^{k+\beta}\right) d y+\left(K+K^{\prime}\right) \int_{Y}|f-g| \varphi d y,
$$

whence $f=g$ thanks to the Gronwall lemma.

We may finally define the notion of strong solution used in the rest of this work. We say that $f$ is a strong solution if $f$ is a weak solution, such that $C(f)$ and $L f$ are functions, and satisfies a uniqueness principle. The spaces we use to look for strong solutions are denoted by $Y=Y_{\mathrm{c}}$ for the coagulation equation, $Y=Y_{\mathrm{f}}$ for the fragmentation equation and $Y=Y_{\mathrm{cf}}$ for the coagulation-fragmentation equation, where

$$
Y_{\mathrm{c}}:=\dot{L}_{2 \alpha}^{1} \cap \dot{L}_{\max (2 \beta, 1)}^{1}, \quad Y_{\mathrm{f}}:=\dot{L}_{\gamma+1}^{1} \cap \dot{L}_{1}^{1}, \quad Y_{\mathrm{cf}}:=\dot{L}_{2 \alpha}^{1} \cap \dot{L}_{\max (2 \beta, \gamma+1,1)}^{1} .
$$

We end this section with a straightforward consequence of the uniqueness Theorem 2.9 and the stability Theorem 2.7.

Corollary 2.10. Suppose that $Y$ is a Banach space such that $Y^{\prime} \subset Y$ with weakly compact injection. Consider a sequence $\left(f^{n}\right)$ of strong solutions to the growth equation (1.14), (1.15). Assume that $\left(f^{n}\right)$ is bounded in $L^{\infty}\left(0, T ; Y^{\prime}\right)$ and that $f^{n}(0,.) \rightarrow f_{\text {in }}$ weakly in $Y$. Then there exists $f \in C([0, T] ; Y)$ such that $f^{n} \rightarrow f$ weakly in $L^{\infty}(0, T ; Y)$, and $f$ is the strong solution to the growth equation (1.14), (1.15) associated to $f_{\text {in. }}$. 


\section{Self-similarity for the fragmentation equation}

We consider in this section the fragmentation equation

$$
\partial_{t} f=L f, \quad f(0, .)=f_{\text {in }},
$$

where the fragmentation operator $L$ is given by (1.4) and the fragmentation rate by (1.5)-(1.6). We assume furthermore

$$
\gamma>-1, \quad B \in \dot{M}_{m}^{1}(0,1) \quad \text { with } m<1, \quad \text { and } \quad B>0,
$$

and, for part of the result,

$$
B \in \dot{B} V_{1}(0,1) \cap \dot{M}_{m}^{1}(0,1) \quad \text { with } m \leqslant \gamma+1 .
$$

Our aim is to study the long time asymptotic behavior of the solution $f$ to the Cauchy problem (3.1) for initial data with finite mass $\int_{0}^{\infty} y f_{\text {in }}(y) d y=\rho>0$. We establish that there exists a unique self-similar solution $F_{\rho}$ of (3.1) with mass $\rho$ and that the behavior of $f(t,$.$) when t \rightarrow \infty$ is described by $F_{\rho}(t,$.$) .$

It is well known that under the condition (1.5)-(1.6) the fragmentation equation (3.1) has a global solution which preserves the total mass, see for instance [22] and the references therein. That means that there is no mass loss in finite time due to the appearance of dust phase (see for instance [7,25], for a description of that singular phenomena). We then look for self-similar solutions to Eq. (3.1) of the form

$$
f(t, y)=t^{2 v} g\left(t^{v} y\right),
$$

in such a way that the total mass is constant in time

$$
\int_{0}^{\infty} f(t, y) y d y=\int_{0}^{\infty} g(y) y d y \equiv \rho, \quad \forall t \geqslant 0 .
$$

On the one hand, introducing the auxiliary variable $z:=y t^{\nu}$, we obtain

$$
\partial_{t} f=v t^{2 v-1}\left(2 g(z)+z g_{z}(z)\right)=v t^{2 v-1} D g(z) .
$$

On the other hand, making the change of variables $y^{\prime} \rightarrow y_{*}:=t^{\nu} y^{\prime}$, we obtain

$$
\begin{aligned}
L f(t, y) & =t^{2 v} \int_{y}^{\infty}\left(y^{\prime}\right)^{\gamma} B\left(y / y^{\prime}\right) g\left(t^{\nu}=y^{\prime}\right) d y^{\prime}-t^{2 v} \int_{0}^{y} \frac{y^{\prime}}{y} y^{\gamma} B\left(y^{\prime} / y\right) d y^{\prime} g\left(t^{\nu} y\right) \\
& =t^{\nu} \int_{t^{\nu} y}^{\infty}\left(t^{-v} y_{*}\right)^{\gamma} B\left(t^{\nu} y / y_{*}\right) g\left(y_{*}\right) d y_{*}-t^{\nu} \int_{0}^{t^{y}} \frac{y_{*}}{t^{\nu} y} y^{\gamma} B\left(y_{*} / t^{\nu} y\right) d y_{*} g\left(t^{\nu} y\right) \\
& =t^{\nu(1-\gamma)} \operatorname{Lg}(z) .
\end{aligned}
$$

Therefore, $f$ is a (self-similar) solution to the fragmentation equation (3.1) if we choose $2 v-1=v(1-\gamma)$, which implies

$$
v:=\frac{1}{1+\gamma}>0,
$$

and if the self-similar profile $g$ is a solution to the "self-similar profile fragmentation" equation already introduced in Section 1:

$$
D g(y)-(1+\gamma) F(g)(y)=0 .
$$


Let us then introduce the so-called "self-similar fragmentation" evolution equation

$$
\partial_{t} g=Q_{\mathrm{SSF}}(g):=-D g+(1+\gamma) L g .
$$

The evident but fundamental remark is that a self-similar profile $g$ is nothing but a stationary solution to (3.9). We may state now the main result of this section.

Theorem 3.1. Assume (1.5)-(1.6), (3.2).

1. For any $\rho>0$ there exists a unique solution $G_{\rho}$ in $\dot{M}_{1}^{1}$ to the self-similar profile fragmentation equation (3.8) such that $M_{1}\left(G_{\rho}\right)=\rho$. Moreover

$$
G_{\rho} \in X_{\infty}:=\bigcap_{k \geqslant m} \dot{M}_{k}^{1}
$$

2. For any $f_{\text {in }} \in Y:=\dot{L}_{m}^{1} \cap \dot{L}_{1}^{1}$ with $\rho:=M_{1}\left(f_{\text {in }}\right)$, there exists a unique solution $f \in C\left([0, T) ; \dot{L}_{1}^{1}\right) \cap$ $L^{1}\left(0, T ; \dot{L}_{\gamma+2}^{1}\right)$ to the fragmentation equation (3.1), such that $M_{1}(f(t))=\rho$ for any $t \geqslant 0$. The solution $g$ in self-similar variables, defining by (1.18), satisfies

$$
(g(t))_{t \geqslant 1} \text { is uniformly bounded in } \dot{M}_{k}^{1} \forall k \geqslant m .
$$

Theorem 3.2. Assume (1.5)-(1.6), (3.2), (3.3).

1. The self-similar profile $G_{\rho}$ has the regularity property $G_{\rho} \in \dot{B} V_{1}$.

2. For any $f_{\text {in }} \in Y \cap B V_{1}$ the associated solution $g$ in self-similar variables satisfies

$$
(g(t))_{t \geqslant 1} \text { is uniformly bounded in } \dot{B} V_{1} \text {. }
$$

3. Finally, for any $f_{\text {in }} \in Y$, the solution $f$ satisfies the asymptotic behavior

$$
\lim _{t \rightarrow+\infty} \int_{0}^{\infty} y\left|f(t, y)-(1+t)^{\frac{2}{1+\gamma}} G_{\rho}\left(y(1+t)^{\frac{1}{1+\gamma}}\right)\right| d y=0 .
$$

In order to prove Theorems 3.1 and 3.2 we first establish some a priori bounds on the solutions to the self-similar fragmentation equation (3.9). We first recall, for the sake of completeness the following version of the Gronwall's lemma to which we systematically refer in all the following. Its proof is classical and well known, and is skipped.

Lemma 3.3. Let $0 \leqslant u \in C([0, \infty))$ satisfy (in the sense of distributions)

$$
u^{\prime}+k_{1} u^{\theta_{1}} \leqslant k_{2} u^{\theta_{2}}+k_{3}
$$

with $\theta_{1} \geqslant 1, \theta_{2} \geqslant 0, \theta_{2} / \theta_{1}<1, k_{1}>0$ and $k_{2}, k_{3} \geqslant 0$. Then, there exists $C_{0}=C_{0}\left(k_{i}, \theta_{i}\right) \geqslant 0$ such that

$$
\sup _{t \geqslant 0} u(t) \leqslant \max \left(C_{0}, u(0)\right) .
$$

Assume moreover $\theta_{1}>1$. For any $\tau>0$, there exists $C_{\tau}=C_{\tau}\left(k_{i}, \theta_{i}, \tau\right) \geqslant 0$ such that

$$
\sup _{t \geqslant \tau} u(t) \leqslant C_{\tau} .
$$

Lemma 3.4. Consider $g_{\text {in }} \in \Xi:=\dot{L}_{1}^{1} \cap \dot{L}_{k}^{1}$ with $k>1$ such that $M_{1}^{1}\left(g_{\text {in }}\right)=\rho$. For any $\tau \geqslant 0$ there exists $v_{\tau}:=$ $\nu_{\tau}(\rho, a, b, k, \tau)$ such that the two following estimates hold:

$$
\sup _{t \geqslant 0}\|g(t, .)\|_{\Xi} \leqslant \max \left(v_{0},\left\|g_{\text {in }}\right\|_{\Xi}\right)
$$


and

$$
\sup _{t \geqslant \tau}\|g(t, .)\|_{\Xi} \leqslant v_{\tau} \quad \text { for } \tau>0 .
$$

Consider $g_{\text {in }} \in \Xi:=\dot{L}_{k}^{1} \cap \dot{L}_{\ell}^{1}$ with $k<1$ and $\ell:=\max (1, k+(1+\gamma))$. There exists $v_{0}:=v_{0}\left(\left\|f_{\text {in }}\right\|_{\dot{L}_{k+\gamma+1} \cap \dot{L}_{\ell}}, a\right.$, $b, k, \tau)$ such that estimate (3.15) holds.

Consider $g_{\text {in }} \in \Xi:=B V_{1} \cap \dot{L}_{\gamma+2}^{1} \cap \dot{L}_{\min (1, \gamma+1)}^{1}$. There exists $v_{0}:=v_{0}\left(\left\|f_{\text {in }}\right\|_{\dot{L}_{\gamma+2}^{1} \cap \dot{L}_{\min (1, \gamma+1)}^{1}}, a, b\right)$ such that estimate (3.15) holds.

As a consequence, for any $\rho>0$, there exists an increasing sequence $\left(\mu_{k}\right)_{k \geqslant \gamma+2}$ such that the sets

$$
\mathcal{X}_{k}:=\left\{g \in X_{k}, M_{1}(g)=\rho,\|g\|_{X_{k}} \leqslant \mu_{k}\right\} \quad \text { and } \quad \mathcal{X}_{\infty}:=\bigcap_{k \geqslant \gamma+2} \mathcal{X}_{k}
$$

are invariant under the flow of the self-similar fragmentation equation (3.9) when (3.2) holds; and the family of sets

$$
\mathcal{Z}_{k}:=\mathcal{X}_{k} \cap\left\{g \in \dot{B} V_{1},\|g\|_{B V_{1}} \leqslant \mu_{0}\right\} \quad \text { and } \quad \mathcal{Z}_{\infty}:=\bigcap_{k \geqslant \gamma+2} \mathcal{Z}_{k}
$$

are invariant when (3.2)-(3.3) hold. In both case, we define $X_{k}:=\dot{M}_{m}^{1} \cap \dot{M}_{k}^{1}$, with the corresponding value of $m$.

Proof of Lemma 3.4. We first recall that for any solution $g$ to (3.9) and any $\phi \in C^{1}$, the following identity holds

$$
\frac{d}{d t} \int_{0}^{\infty} g \phi d y=\int_{0}^{\infty} g\left((y \phi)_{y}-2 \phi\right) d y+(1+\gamma) \int_{0}^{\infty} g y^{\gamma} \int_{0}^{y} B\left(y^{\prime} / y\right)\left(\phi^{\prime}-\frac{y^{\prime}}{y} \phi\right) d y^{\prime} d y .
$$

Taking $\phi=y^{k}$, we get

$$
\frac{d}{d t} M_{k}=(k-1) M_{k}+C_{k, \gamma}(1-k) M_{\gamma+1+k},
$$

with $C_{k, \gamma}=\frac{1+\gamma}{1-k} \int_{0}^{1} B(\sigma)\left(\sigma^{k}-\sigma\right) d \sigma>0$. We deduce first, for $k=1$, the conservation of mass

$$
\frac{d}{d t} M_{1}=0 \quad \text { and } \quad M_{1}(t) \equiv M_{1}(0)=\rho .
$$

Next, for $k>1$, since, by Holder's inequality, $M_{k} \leqslant M_{1}^{1-\theta} M_{\gamma+1+k}^{\theta}$ with $\theta \in(0,1)$, we deduce from (3.19)

$$
\frac{d}{d t} M_{k} \leqslant(k-1) M_{k}-\kappa M_{k}^{1 / \theta}
$$

with $\kappa=\kappa(k, \gamma, \rho)>0$ and we conclude (3.15) and (3.16) with $\Xi=\dot{L}_{1}^{1} \cap \dot{L}_{k}^{1}$ thanks to the Gronwall's Lemma 3.3.

Finally, for $k<1$, let us write (3.19) as,

$$
\frac{d}{d t} M_{k}+(1-k) M_{k}=C_{k, \gamma}(1-k) M_{\gamma+1+k} .
$$

In the one hand, if $1 \leqslant \gamma+1+k$ and then $g_{\text {in }} \in L_{\gamma+1+k}^{1}$ by assumption, we deduce from (3.20), the estimates already obtained for $k>1$, and Gronwall's Lemma 3.3 that (3.15) holds with $\Xi=\dot{L}_{\gamma+1+k}^{1} \cap \dot{L}_{k}^{1}$. On the other hand, if $\gamma+1+k<1$, we deduce from the Young inequality $M_{\gamma+1+k} \leqslant \varepsilon M_{k}+C_{\varepsilon} M_{1}($ remind that $\gamma+1>0)$ that

$$
\frac{d}{d t} M_{k}+\frac{1-k}{2} M_{k}=C_{k, \gamma}^{\prime} M_{1}
$$

for some positive constant $C_{k, \gamma}^{\prime}$, and we conclude as above that (3.15) holds with $\Xi=\dot{L}_{1}^{1} \cap \dot{L}_{k}^{1}$. 
In order to obtain the $\dot{B} V_{1}$ estimate, we differentiate the self-similar fragmentation equation (3.9) and we obtain

$$
\partial_{t} g_{y}=-g_{y}-D\left(g_{y}\right)+(L g)_{y},
$$

with

$$
(L g)_{y}=-2 b(y, y) g+\int_{y}^{\infty} \partial_{2} b\left(y^{\prime \prime}, y\right) g^{\prime \prime} d y^{\prime \prime}+\int_{0}^{y} \frac{y^{\prime}}{y}\left(\frac{b\left(y, y^{\prime}\right)}{y}-\partial_{1} b\left(y, y^{\prime}\right)\right) d y^{\prime} g-\int_{0}^{y} \frac{y^{\prime}}{y} b\left(y, y^{\prime}\right) d y^{\prime} g_{y} .
$$

As a consequence, we get

$$
\frac{d}{d t} \int_{0}^{\infty}\left|g_{y}\right| y d y \leqslant-\int_{0}^{\infty}\left|g_{y}\right|\left(y+B_{1} y^{2+\gamma}\right) d y+C_{B} \int_{0}^{\infty} g y^{\gamma+1} d y \leqslant-\int_{0}^{\infty} y\left|g_{y}\right| d y+C_{B} M_{\gamma+1}(t)
$$

with $C_{B}=(2+\gamma) B_{1}+B_{2}^{\prime}+2 B(1), B_{1}=\int_{0}^{1} z B(z) d z, B_{1}^{\prime}=\int_{0}^{1} z\left|B^{\prime}(z)\right| d z$. We conclude, again by the Gronwall's Lemma 3.3 that (3.15) holds when $\Xi=\dot{B} V_{1} \cap \dot{L}_{\gamma+2}^{1} \cap \dot{L}_{\min (1, \gamma+1)}^{1}$.

We present now a contraction property in $\dot{L}_{1}^{1}$ of the self-similar fragmentation operator $Q_{\mathrm{SSF}}$.

Lemma 3.5. For any function $H \in L_{2+\gamma}^{1}$, there holds

$$
D(H):=\int_{0}^{\infty} Q_{\mathrm{SSF}}(H) \operatorname{sign}(H) y d y \leqslant 0 .
$$

Moreover,

$$
D(H)=0 \text { and } \int_{0}^{\infty} y H d y=0 \text { implies } H=0 .
$$

As a consequence, for any $\rho \geqslant 0$, there exists at most one solution $G_{\rho}$ to the stationary self-similar fragmentation equation (3.8) such that $M_{1}\left(G_{\rho}\right)=\rho$.

Proof of Lemma 3.5. Suppose first $H \in C_{c}$. Then, a straightforward calculation gives

$$
D(H):=\int_{0}^{\infty} H \int_{0}^{y} b\left(y, y^{\prime}\right)\left(\phi^{\prime}-\frac{y^{\prime}}{y} \phi\right) d y^{\prime} d y, \quad \phi:=\operatorname{sign}(H) y .
$$

By density this identity holds for all $H \in \dot{L}_{1}^{1} \cap \dot{L}_{2+\gamma}^{1}$. Then, (3.21) follows from the fact:

$$
H\left(\phi^{\prime}-\frac{y^{\prime}}{y} \phi\right)=\left(H \operatorname{sign}\left(H^{\prime}\right)-|H|\right) y^{\prime} \leqslant 0 \quad \forall y, y^{\prime} .
$$

If we suppose now that $D(H)=0$, we deduce

$$
H \operatorname{sign}\left(H^{\prime}\right)=|H| \quad \forall y, y^{\prime} .
$$

As a consequence, $\operatorname{sign} H=\operatorname{sign} H^{\prime} \forall y, y^{\prime}$ or, in other words, sign $H$ is constant on $(0, \infty)$. Therefore if $H \not \equiv 0$ then $\int y H d y \neq 0$ which is a contradiction. 
Proof of Theorem 3.2. Point 2. Assume first that $f_{\text {in }} \in Y_{\ell}:=Y \cap \dot{L}_{\ell}^{1}$ for a given $\ell>\gamma+1$. Let us define $g_{\text {in }} \in Y_{\ell}$ using (1.18) and introduce the truncated fragmentation kernel

$$
b_{n}\left(y, y^{\prime}\right)=\min \left(y^{\gamma}, n\right) B\left(y^{\prime} / y\right) .
$$

By Lemma 2.8, there exists a unique solution $g_{n} \in C([0, T] ; Y)$ to the associated self-similar fragmentation equation (3.9) with initial data $g_{\text {in }}$. Moreover, coming back to the proof of Lemma 3.4, we easily verify that $\left(g_{n}\right)$ satisfies estimates (3.15) and (3.16) uniformly in $n \geqslant 1$. Proceeding along the line of the existence theorem in [27] (which is based on a perturbation of the uniqueness Theorem 2.9 introduced in [43]), we deduce that $\left(g_{n}\right)$ is a Cauchy sequence in $C\left([0, T] ; Y_{\ell}\right)$. Let us denote by $g$ its limit. Then we may pass to the limit in the equation satisfied by $\left(g_{n}\right)$ and we obtain that $g \in C\left([0, T] ; Y_{\ell}\right)$ is a solution to the self-similar fragmentation equation (3.9) with initial data $g_{\text {in }}$ and satisfying (3.10) and (3.11). By the differential inequality established in the uniqueness Theorem 2.9 we may remove the additional assumption $g_{\text {in }} \in Y_{\ell}$. We conclude using (1.16).

Proof of Theorem 3.2. Point 1. We denote by $S$ the semigroup associated to the self-similar fragmentation equation (3.9) built above. On the one hand, the sets $\mathcal{Z}_{k}$ defined in Lemma 3.5 are invariant under the action of $S$ and, of course, $\mathcal{Z}_{k} \subset Y$ with compact injection for the weak sense of convergence in $Y$. On the other hand, by the stability result Theorem 2.7 and the uniqueness result Theorem 2.9, we infer that for any $t>0$ the map $S(t): Y \rightarrow Y$ is weakly sequentially continuous. Therefore, we may apply Theorem 1.2 and we obtain the existence of a selfsimilar profile $G_{\rho} \in \mathcal{Z}_{k}$ with mass $\rho$. Finally, $G_{\rho}$ is unique by to the contraction property Lemma 3.5. Since $k$ may be taken arbitrary large, we finally obtain $G_{\rho} \in \mathcal{Z}_{\infty}$.

Proof of Theorem 3.2. Point 2. Let us prove now (3.12). To this end we consider $f_{\text {in }} \in C_{c}^{1}\left(\mathbb{R}_{+}\right)$, such that $M_{1}\left(f_{\text {in }}\right)=\rho$ and $f_{\text {in }} \neq G_{\rho}$ (since otherwise there is nothing to be proved). Let $g=S(\tau) f_{\text {in }}$ be the unique corresponding solution to (3.9) such that $g(0)=f_{\text {in. }}$. Let us show that

$$
H_{\rho}(g(\tau))=\int_{0}^{\infty} y\left|g(\tau, y)-G_{\rho}(y)\right| d y,
$$

is a strict Lyapunov functional on $\dot{L}_{1}^{1}$. To this end, we first notice, using the equation and introducing $h(\tau, y)=$ $g(\tau, y)-G_{\rho}(y)$, that

$$
\frac{d}{d t} H_{\rho}(g(\tau))=D(h(\tau)) \leqslant 0 .
$$

This shows that $H_{\rho}(g(t))$ is nonincreasing. In order to prove that it is actually strictly decreasing, suppose that there exists two different instants, $0<\tau_{1}<\tau_{2}$ such that $H_{\rho}\left(g\left(\tau_{1}\right)\right)=H_{\rho}\left(g\left(\tau_{2}\right)\right)$. Then,

$$
\int_{\tau_{1}}^{\tau_{2}} D(H(\tau)) d t=0 .
$$

Since $D(H(\tau)) \leqslant 0$ for all $\tau>0$ we deduce that $D(H(\tau))=0$ for $\tau \in\left(\tau_{1}, \tau_{2}\right)$. Since $M_{1}(H)=0$ for all $\tau$ we deduce, by Lemma 3.5, that $H(\tau)=0$ for all $\tau \in\left(\tau_{1}, \tau_{2}\right)$. But this implies $H \equiv 0$ which is absurd.

On the other hand, since $f_{\text {in }} \in \dot{L}_{m}^{1} \cap \dot{B} V_{1} \dot{L}_{\gamma+2}^{1}$ for any $k \geqslant 2$, Lemma 3.4 implies that $(g(\tau))_{\tau>0}$ is bounded in $\dot{L}_{m}^{1} \cap \dot{B} V_{1} \dot{L}_{\gamma+2}^{1}$. The trajectory $(g(\tau))_{\tau>0}$ belongs then to a compact subset of $\dot{L}_{1}^{1}$. We conclude by the Lasalle's invariance principle (see for instance [11]), that

$$
\lim _{\tau \rightarrow+\infty} H_{\rho}(g(\tau))=0 .
$$

Using a classical density argument and the contraction property of $S(\tau)$, we deduce that (3.23) holds for all initial data $f_{\text {in }} \in Y$. 
Consider now the function $f$ defined by (1.16). By construction, $f \in C\left([0,+\infty) ; \dot{L}_{1}^{1}\right), M_{1}(f(t))=\rho$. It is a straightforward calculation to check that this function $f$ satisfies

$$
\partial_{t} f=L f, \quad f(0, y)=f_{\text {in }} .
$$

The uniqueness of this solution in $C\left([0,+\infty) ; \dot{L}_{1}^{1}\right)$ is given by Theorem 2.9. The regularity properties of $S(\tau) f_{\text {in }}$ give the corresponding properties for the function $f$. Finally, by (3.4), we obtain

$$
\int_{0}^{\infty} y\left|f(t, y)-(1+t)^{\frac{2}{1+\gamma}} G_{\rho}\left(y(1+t)^{\frac{1}{1+\gamma}}\right)\right| d y=H_{\rho}(g)
$$

and (3.12) follows thanks to (3.23).

Proof of Theorem 3.1. The proof of Theorem 3.1 follows exactly along the same lines as the proof of Theorem 3.2. We then skip it.

Remark 3.6. To prove the existence part in Theorem 3.1 one may also use the stability result Theorem 2.7 , introducing the regularized fragmentation kernel

$$
b_{\varepsilon}\left(y, y^{\prime}\right)=y_{\varepsilon}^{\gamma} B_{\varepsilon}\left(y^{\prime} / y\right), \quad y_{\varepsilon}=\min \left(0, y-\varepsilon, \frac{2}{\varepsilon}-y\right), \quad B_{\varepsilon}:=\left(B \chi_{\varepsilon}\right) *_{z} \rho_{\varepsilon},
$$

where $0 \leqslant \chi_{\varepsilon}, \rho_{\varepsilon} \in \mathcal{D}(\mathbb{R})$, supp $\chi_{\varepsilon} \subset(2 \varepsilon, 1]$, supp $\rho_{\varepsilon} \subset(-\varepsilon, \varepsilon), \chi_{\varepsilon} \nearrow 1$ uniformly and $\rho_{\varepsilon} \rightarrow \delta_{0}$ in $\mathcal{D}^{\prime}(\mathbb{R})$.

\section{Stationary solutions for the coagulation-fragmentation model}

In this section we consider the coagulation-fragmentation equation

$$
\partial_{t} f=Q(f)=C(f)+L f, \quad f(0, .)=f_{\text {in }},
$$

where the fragmentation and the coagulation operator $L$ and $C$ are defined by (1.4), (1.7) with fragmentation and coagulation rates given by (1.5), (1.6) and (1.8). We furthermore assume that

$$
(\beta, \gamma) \neq(1,-1), \quad(\alpha, \gamma) \neq(0,-1) \quad \text { and } \quad B \in \dot{M}_{m}^{1} \quad \text { with } m \leqslant 2 \alpha .
$$

We will also need the following additional assumption

$$
B \in L^{\infty} \text {. }
$$

Our main result is the existence of stationary solution to the coagulation-fragmentation equation (4.1) for any given mass $\rho>0$.

Theorem 4.1. 1. Assume that $a$ and $b$ satisfy (1.5), (1.6), (1.8), (4.2). For any $\rho>0$ there exists at least one weak stationary solution $F_{\rho} \in X_{\infty}$ to the coagulation-fragmentation equation (4.1) such that $M_{1}\left(F_{\rho}\right)=\rho$, where

$$
X_{\infty}:=\bigcap_{k \geqslant 1} X_{k} \quad \text { with } X_{k}:=\dot{M}_{2 \alpha}^{1} \cap \dot{M}_{k}^{1} \text { if } \alpha=0, \quad X_{k}:=\dot{M}_{m}^{1} \cap \dot{M}_{k}^{1} \text { if } \alpha<0 .
$$

Assuming moreover (4.3), we may choose the stationary solution such that $F_{\rho} \in Y_{\infty}$ where

$$
Y_{\infty}:=\bigcap_{k \geqslant 1} Y_{k}, \quad Y_{k}:=L^{k+1} \cap X_{k} .
$$


2. Assume (4.2) and $f_{\text {in }} \in Y:=\dot{L}_{2 \alpha}^{1} \cap \dot{L}_{\max (2 \beta, 2-\beta, \gamma+1)}^{1}$. Then there exists a unique strong solution $f \in$ $C\left([0, T) ; \dot{L}_{1}^{1}\right) \cap L^{\infty}(0, T ; Y) \forall T>0$ to the coagulation-fragmentation equation (4.1). Moreover, $f$ satisfies

$$
(f(t))_{t \geqslant 1} \text { is uniformly bounded in } X_{k} \forall k \geqslant 1 \text {. }
$$

Assume moreover (4.3) and $f_{\text {in }} \in L^{p}, p \geqslant 2$. Then $f$ also satisfies

$$
(f(t))_{t \geqslant 0} \text { is uniformly bounded in } L^{p} .
$$

We start with some a priori estimates on the solutions to the $\mathrm{CF}$ equation.

Lemma 4.2. Assume $(\beta, \gamma) \neq(1,-1)$ and $B \in \dot{M}_{\lambda}^{1}$. There exists a constant $v_{0}:=v_{0}(\rho, a, b) \geqslant 0$ such that for any $f_{\text {in }} \in \Xi_{0}:=\dot{L}_{\lambda}^{1} \cap \dot{L}_{2-\beta}^{1}$ the global estimate (3.15) holds.

Consider now $f_{\text {in }} \in \Xi:=\Xi_{0} \cap \dot{L}_{k}^{1}$ with $k>2-\beta$. For any $\tau \geqslant 0$, there exists $v_{\tau}=v_{\tau}\left(\left\|f_{\text {in }}\right\| \Xi_{0}, a, b, k\right)$ such that the global estimates (3.15) and (3.16) hold.

Proof of Lemma 4.2. For any $k \in \mathbb{R}$, let us define

$$
\Lambda_{k}\left(y, y^{\prime}\right):=(k-1)\left(y^{\alpha}\left(y^{\prime}\right)^{\beta}+y^{\beta}\left(y^{\prime}\right)^{\alpha}\right)\left(\left(y+y^{\prime}\right)^{k}-y^{k}-\left(y^{\prime}\right)^{k}\right) \geqslant 0,
$$

and denote $z=y^{\prime} / y$. On the one hand, for $k>1$ and $y>y^{\prime}$, we have

$$
\begin{aligned}
\Lambda_{k}\left(y, y^{\prime}\right) & =(k-1) y^{\lambda+k}\left(z^{\alpha}+z^{\beta}\right)\left((1+z)^{k}-z^{k}-1\right) \\
& \leqslant C_{k} y^{\lambda+k} z^{1+\alpha} \leqslant C_{k}\left[y^{\beta-1+k}\left(y^{\prime}\right)^{1+\alpha}+\left(y^{\prime}\right)^{\beta-1+k} y^{1+\alpha}\right],
\end{aligned}
$$

for a constant $C_{k}>0$, and therefore, the same holds for any $y, y^{\prime} \geqslant 0$. We deduce that for $k>1$

$$
\frac{d}{d t} M_{k} \leqslant C_{k, 1} M_{\beta-1+k} M_{1+\alpha}-C_{k, 2} M_{1+\gamma+k}
$$

for some constants $C_{k, i}>0$. On the other hand, for $y>y^{\prime}$, and using that $\lambda<1$, we have

$$
\Lambda_{\lambda}\left(y, y^{\prime}\right)=(1-\lambda) y^{\lambda+\lambda}\left(z^{\alpha}+z^{\beta}\right)\left(z^{\lambda}+1-(1+z)^{\lambda}\right) \geqslant C_{\lambda} y^{\lambda+\lambda} z^{\lambda} \geqslant C_{\lambda} y^{\lambda}\left(y^{\prime}\right)^{\lambda},
$$

for a constant $C_{\lambda}>0$ and therefore, the same holds for any $y, y^{\prime} \geqslant 0$. We deduce that

$$
\frac{d}{d t} M_{\lambda} \leqslant C_{\lambda, 1} M_{1+\gamma+\lambda}-C_{\lambda, 2} M_{\lambda}^{2}
$$

for some constants $C_{\lambda, i}>0$. Gathering (4.7) and (4.8) we find

$$
\begin{aligned}
\frac{d}{d t}\left(M_{\lambda}+M_{2-\beta}\right) & \leqslant C_{2-\beta, 1} M_{1} M_{1+\alpha}+C_{\lambda, 1} M_{1+\gamma+\lambda}-C_{\lambda, 2} M_{\lambda}^{2}-C_{2-\beta, 2} M_{1+\gamma+2-\beta} \\
& \leqslant C_{2-\beta, 1} M_{1}\left(M_{1}+M_{\lambda}\right)+C_{\lambda, 1} K_{\varepsilon} M_{\lambda}-C_{\lambda, 2} M_{\lambda}^{2}+\left(\varepsilon C_{\lambda, 1}-C_{2-\beta, 2}\right) M_{1+\gamma+2-\beta}
\end{aligned}
$$

for a constant $K_{\varepsilon}$ for any $\varepsilon>0$, where we have use twice the Young inequality and the fact that $1+\alpha \in[\lambda, 1]$ and $1+\gamma+\lambda \in[\lambda, 1+\gamma+2-\beta)$.

For $\varepsilon>0$ small enough and using the Holder inequalities $M_{2-\beta} \leqslant M_{1}^{1-\theta} M_{1+\gamma+2-\beta}^{\theta}$ with $\theta \in(0,1]$, we obtain

$$
\frac{d}{d t}\left(M_{\lambda}+M_{2-\beta}\right) \leqslant C_{1}+C_{2} M_{\lambda}-C_{3} M_{\lambda}^{2}-C_{4} M_{2-\beta}^{p}
$$

for some constants $C_{i}=C_{i}(\rho)>0$ and $p=1 / \theta$ (if $\beta<1$ ) or $p=1$ (if $\beta=1$ ). Therefore, using a straightforward variant of the Gronwall's Lemma 3.3, we deduce (3.15) for some positive constant $v=v(\rho, a, b)$. 
On the other hand, for $k>2-\beta$, we come back to (4.7). Using $M_{1+\alpha} \leqslant M_{\lambda}+M_{1}$, the Holder inequalities $M_{\beta-1+k} \leqslant M_{1}^{1-\theta_{1}} M_{k}^{\theta_{1}}, M_{k}^{\theta_{2}} \leqslant M_{1}^{1 / \theta_{2}-1} M_{k+1+\gamma}$, with $\theta_{1} \in[0,1], \theta_{2} \geqslant 1$ and $\theta_{2} / \theta_{1}>1$ (due to condition (4.2)) we obtain

$$
\frac{d}{d t} M_{k} \leqslant C_{1} M_{k}^{\theta_{1}}-C_{2} M_{k}^{\theta_{2}}
$$

for some constants $C_{i}=C_{i}(\rho)>0$. Using again the Gronwall's Lemma 3.3 we conclude that (3.15) and (3.16) holds for $k>2-\beta$.

We prove now some more a priori estimates on solutions to Eq. (4.1) under additional assumptions on the fragmentation and the coagulation rate functions.

Lemma 4.3. Assume $(\alpha, \gamma) \neq(0,-1)$ and $B \in \dot{M}_{k}^{1}$ with $k \leqslant 0$. Consider $f_{\text {in }} \in \Xi:=\Xi_{0} \cap \dot{L}_{k}^{1}$ with $\Xi_{0}:=\dot{L}_{k}^{1} \cap$ $\dot{L}_{\max (1+\gamma+k, 2-\beta)}^{1}$. There exists a constant $v_{0}:=v_{0}\left(\|f\|_{\Xi_{0}}, a, b, k\right) \geqslant 0$ such that the global estimate (3.15) holds. Assume moreover $\alpha<0$, but just $f_{\text {in }} \in \Xi_{0}$. For any $\tau>0$, there exists a constant $v_{\tau}:=v_{\tau}\left(\|f\|_{\Xi_{0}}, a, b, k\right) \geqslant 0$ such that the global estimates (3.16) hold.

Assume $(\alpha, \gamma) \neq(0,-1)$ and $B \in L^{\infty}$. Consider $f_{\text {in }} \in \Xi:=\Xi_{0} \cap L^{p}$ with $\Xi_{0}:=\dot{L}_{\min (\gamma, 0)}^{1} \cap \dot{L}_{\max (1+\gamma, 2-\beta)}^{1}$ and $p \geqslant 2$. Then, there exists a constant $v_{0}:=v_{0}\left(\|f\| \Xi_{0}, a, b\right) \geqslant 0$ such that the global estimate (3.15) holds.

Proof of Lemma 4.3. First, for $k \leqslant 0$, we have $\Lambda_{k}\left(y, y^{\prime}\right) \leqslant-y^{\alpha+k}\left(y^{\prime}\right)^{\beta}$ and thus

$$
\frac{d}{d t} M_{k} \leqslant C_{k, 1} M_{1+\gamma+k}-\frac{1}{2} M_{\alpha+k} M_{\beta} \leqslant C_{1} M_{k}^{\theta_{1}}-C_{2} M_{k}^{\theta_{2}},
$$

with $\theta_{1} \in[0,1), \theta_{2} \geqslant 1, C_{i}>0$, where we have used the Holder inequalities $M_{1+\gamma+k} \leqslant M_{k}^{1-\theta_{1}} M_{\max (1+\gamma+k, \lambda)}^{\theta_{1}}$, $M_{k} \leqslant M_{1}^{1-\theta_{1}} M_{\alpha+k}^{\theta_{1}}$ and the fact that $M_{\beta}$ and $M_{\max (1+\gamma+k, \lambda)}$ can be bounded using to Lemma 4.2. Estimates (3.15) and (3.16) with $\Xi=\dot{L}_{k}^{1}$ follow from (4.9) and Lemma 3.3.

We now prove the $L^{p}$ estimate. On the one hand, we have

$$
\begin{aligned}
\left\langle C(f), f^{p-1}\right\rangle & =\int_{0}^{\infty} \int_{0}^{\infty} y^{\alpha}\left(y^{\prime}\right)^{\beta} f f^{\prime}\left(\left(f^{\prime \prime}\right)^{p-1}-f^{p-1}-\left(f^{\prime}\right)^{p-1}\right) d y d y^{\prime} \\
& \leqslant \int_{0}^{\infty} \int_{0}^{\infty} f y^{\alpha}\left(y^{\prime}\right)^{\beta}\left(\frac{\left(f^{\prime}\right)^{p}}{p}+\frac{\left(f^{\prime \prime}\right)^{p}}{p^{\prime}}\right) d y d y^{\prime}-M_{\beta} \int_{0}^{\infty} f^{p} y^{\alpha} d y-M_{\alpha} \int_{0}^{\infty}\left(f^{\prime}\right)^{p}\left(y^{\prime}\right)^{\beta} d y^{\prime} \\
& \leqslant \frac{1}{p^{\prime}} \int_{0}^{\infty} \int_{0}^{\infty} f y^{\alpha}\left(f^{\prime}\right)^{p}\left[\left(y^{\prime}-y\right)^{\beta} \mathbf{1}_{y^{\prime}>y}-\left(y^{\prime}\right)^{\beta}\right] d y d y^{\prime}-M_{\beta} \int_{0}^{\infty} f^{p} y^{\alpha} d y \\
& \leqslant-M_{\beta} \int_{0}^{\infty} f^{p} y^{\alpha} d y
\end{aligned}
$$

where we have used the Young inequality and the change of variables $\left(y, y^{\prime}\right) \rightarrow\left(y, y^{\prime \prime}=y+y^{\prime}\right)$. On the other hand, we have

$$
\left\langle L f, f^{p-1}\right\rangle=\int_{0}^{\infty} f y^{\gamma} \int_{0}^{y} B\left(y^{\prime} / y\right)\left[\left(f^{\prime}\right)^{p-1}-\frac{y^{\prime}}{y} f^{p}\right] d y^{\prime} d y
$$




$$
\leqslant\|B\|_{L^{\infty}} M_{\gamma} \int_{0}^{\infty} f^{p-1} d y-\|B\|_{\dot{L}_{1}^{1}} \int_{0}^{\infty} f^{p} y^{\gamma+1} d y .
$$

Putting together these two bounds and noticing that $\alpha \leqslant 0 \leqslant \gamma+1$ and that

$$
\int_{0}^{\infty} f^{p-1} d y \leqslant\left(\int_{0}^{\infty} f d y\right)^{\frac{1}{p-1}}\left(\int_{0}^{\infty} f^{p} d y\right)^{\frac{p-2}{p-1}}
$$

(Holder's inequality), we obtain

$$
\begin{aligned}
\frac{d}{d t} \int_{0}^{\infty} \frac{f^{p}}{p} d y & =\left\langle C(f), f^{p-1}\right\rangle+\left\langle L f, f^{p-1}\right\rangle \\
& \leqslant\|B\|_{L^{\infty}} M_{\gamma} M_{0}^{\frac{1}{p-1}}\left(\int_{0}^{\infty} f^{p} d y\right)^{\frac{p-2}{p-1}}-\min \left(\|B\|_{\dot{L}_{1}^{1}}, M_{\beta}\right) \int_{0}^{\infty} f^{p} d y .
\end{aligned}
$$

Notice that $M_{0}$ and $M_{\gamma}$ have been bounded by above in the preceding step or in Lemma 4.2 (because $B \in L^{\infty}$ implies $B \in \dot{M}_{\min (\gamma, 0)}^{1}$ ) and that $M_{\beta}$ may be bounded by below in the following way $M_{\beta} \geqslant M_{1}^{2-\beta} M_{2}^{\beta-1}$ using the Holder inequality and Lemma 4.2 in order to estimate (by above) $M_{2}$. Finally, we conclude that (3.15) holds for $\Xi=L^{p}$ thanks to the Gronwall's Lemma 3.3.

Remark 4.4. Lemma 4.3 extends to the coagulation-fragmentation model a uniform $L^{p}$ a priori bound which was already known to be true for the coagulation equation [34,42].

Proof of Theorem 4.1. Point 2. Consider the truncated fragmentation kernel (3.22) and the truncated coagulation kernel:

$$
a_{n}\left(y, y^{\prime}\right):=\left(y^{n}\right)^{\alpha}\left(y_{n}^{\prime}\right)^{\beta}+\left(y^{\prime n}\right)^{\alpha}\left(y_{n}\right)^{\beta}, \quad y_{n}=\min (y, n), y^{n}=\max (y, 1 / n),
$$

we may easily proceed as in the proof of Theorem 3.1 and conclude to the existence of a unique solution to the Cauchy problem owning the properties as stated in Theorem 4.12 . We refer again to $[34,33,22,36]$ for more details.

Proof of Theorem 4.1. Point 1. Assume first (1.5), (1.6), (1.8), (4.2), (4.3) and let denote by $S$ the semigroup associated to the coagulation-fragmentation equation. Then, $S: Y \rightarrow Y$ and, for well chosen constants $\mu_{k}, S: \mathcal{A}_{k} \rightarrow \mathcal{A}_{k}$ where

$$
\mathcal{A}_{k}:=\left\{f \in L^{1}, M_{1}(f)=\rho,\|f\|_{L^{2}} \leqslant \mu_{0},\|f\|_{\dot{L}_{2 \alpha}^{1} \cap \dot{L}_{k}^{1}} \leqslant \mu_{k}\right\} \quad \forall k \geqslant \max (\gamma+1,2) .
$$

Next, for any integer $\ell \geqslant 2$ we define $\mathcal{Z}_{\ell}=\bigcap_{k=2, \ldots, \ell} \mathcal{A}_{k}$, so that $S: \mathcal{Z}_{\ell} \rightarrow \mathcal{Z}_{\ell}$ and $\left(\mathcal{Z}_{\ell}\right)$ is a decreasing sequence of sets. Following step by step the proof of Theorem 3.1, we prove the existence of a stationary solution $F_{\rho, \ell} \in \mathcal{Z}_{\ell}$ to the coagulation-fragmentation equation, that is $Q\left(F_{\rho, \ell}\right)=0$ for any integer $\ell \geqslant 2$. By compactness, there exists a subsequence $\left(F_{\rho, \ell_{n}}\right)$ of $\left(F_{\rho, \ell}\right)$ and a function $F_{\rho}$ of mass $\rho$, such that $F_{\rho, \ell_{n}} \rightarrow F_{\rho}$ weakly in any $\mathcal{Z}_{m}$ when $n$ goes to $\infty$, for any integer $m \geqslant 2$. We conclude that $F_{\rho}$ satisfies the properties stated in Theorem 4.1 thanks to the stability principle Theorem 2.7 .

Suppose finally that we do not make the assumption (4.3). By regularisation, we may find a sequence $\left(B^{\varepsilon}\right)$ such that $B^{\varepsilon} \in L^{\infty}$ and $B^{\varepsilon} \rightarrow B$ weakly in $\dot{M}_{m}^{1}$. Using the preceding step, we infer that, for any $\rho>0$ and $\varepsilon>0$, there exists a stationary solution $F_{\rho}^{\varepsilon} \in \mathcal{Z}_{\infty}$ to the regularized coagulation-fragmentation equation, that is $Q_{\varepsilon}\left(F_{\rho}^{\varepsilon}\right)=0$, $M_{1}\left(F_{\rho}^{\varepsilon}\right)=\rho$. From Lemmas 4.2 and 4.3 , the sequence $\left(F_{\rho}^{\varepsilon}\right)$ is bounded in $X_{k}$ for any $k \geqslant 2-\beta$, and therefore, 
there exists $F_{\rho} \in X_{\infty}$ and a subsequence $\left(F_{\rho}^{\varepsilon^{\prime}}\right)$ such that $F_{\rho}^{\varepsilon^{\prime}} \rightarrow F_{\rho}$ weakly in $X_{k}$ for any integer $k \geqslant 2$. Again, we conclude that $F_{\rho}$ is a weak stationary solution with mass $\rho$ to the coagulation-fragmentation equation thanks to the stability principle Theorem 2.7 .

\section{Self-similarity for the coagulation equation}

We consider in this section the coagulation equation

$$
\partial_{t} f=C(f), \quad f(0, .)=f_{\text {in }},
$$

where the coagulation operator is given by (1.7) and the homogeneous coagulation frequency is given by (1.8). We make the additional assumption

$$
\beta<1 .
$$

Our main result is the existence of mass preserving self-similar solutions to the coagulation equation (5.1) for any given mass $\rho>0$. Since the solutions to the coagulation equation (5.1) with subcritical coagulation frequency (1.8) are known to conserve the mass $M_{1}(f)$ of the solution for all time (see for instance [37,23]), we look for a self-similar solution $f$ of the form (3.4) with $g \in \dot{L}_{1}^{1}$.

On the one hand, $f$ satisfies (3.5) and (3.6). On the other hand, after the change of variables $y^{\prime} \rightarrow y_{*}:=t^{\nu} y^{\prime}$ and using the scaling property $a\left(\tau y, \tau y_{*}\right)=\tau^{\lambda} a\left(y, y_{*}\right)$, we obtain

$$
\begin{aligned}
C(f)(t, y) & =\frac{t^{4 v}}{2} \int_{0}^{y} a\left(y^{\prime}, y-y^{\prime}\right) g\left(t^{v}\left(y-y^{\prime}\right)\right) g\left(t^{\nu} y^{\prime}\right) d y^{\prime}-t^{4 v} \int_{0}^{\infty} a g\left(t^{\nu} y^{\prime}\right) g\left(t^{\nu} y\right) d y^{\prime} \\
& =\frac{t^{3 v}}{2} \int_{0}^{t^{v} y} a\left(t^{-v} y_{*}, y-t^{-v} y_{*}\right) g\left(t^{\nu} y-y_{*}\right) g_{*} d y_{*}-t^{3 v} \int_{0}^{\infty} a\left(y, t^{-v} y_{*}\right) g_{*} g\left(t^{\nu} y\right) d y_{*} \\
& =\frac{t^{3 v-\lambda v}}{2} \int_{0}^{t^{v} y} a\left(y_{*}, t^{\nu} y-y_{*}\right) g\left(t^{\nu} y-y_{*}\right) g_{*} d y_{*}-t^{3 v-\lambda v} \int_{0}^{\infty} a\left(t^{\nu} y, y_{*}\right) g_{*} g\left(t^{\nu} y\right) d y_{*} \\
& =t^{3 v-\lambda v} C(g)(z),
\end{aligned}
$$

where we have set $z:=y t^{\nu}$. If we choose $3 v-\lambda v=2 v-1$, or equivalently,

$$
v:=-\frac{1}{1-\lambda}<0,
$$

the self-similar profile $g$ satisfies the homogeneous self-similar profile coagulation equation

$$
D g(z)+(1-\lambda) C(g)(z)=0 .
$$

A self-similar profile is therefore a stationary solution of the "self-similar coagulation" evolution equation

$$
\partial_{t} g=D g+(1-\lambda) C(g) .
$$

Theorem 5.1. 1. Suppose $\alpha=0$. Then, for any $\rho>0$ there exists at least one self-similar profile $F_{\rho} \in X_{\infty}$, dual weak solution of the coagulation equation (5.1), (1.8), (5.2) such that $M_{1}\left(F_{\rho}\right)=\rho$, where

$$
X_{\infty}:=\bigcap_{k \geqslant 2-\beta} X_{k}, \quad X_{k}=\dot{M}_{k}^{1} \cap \dot{M}_{\lambda}^{1} .
$$


2. Suppose $\alpha<0$. Then, for any $\rho>0$ there exists at least one self-similar profile $F_{\rho} \in X_{\infty}$, dual weak and mild solution of the coagulation equation (5.1), (1.8), (5.2) such that $M_{1}\left(F_{\rho}\right)=\rho$, where

$$
X_{\infty}:=\bigcap_{k \geqslant 2-\beta} X_{k}, \quad X_{k}:=\dot{M}_{k}^{1} \cap \dot{M}_{-k}^{1} .
$$

3. For any $f_{\mathrm{in}} \in Y:=\dot{L}_{2 \alpha}^{1} \cap \dot{L}_{\max (2 \beta, 2-\beta)}^{1}$, there exists a unique solution $f \in C\left([0, T) ; \dot{L}_{1}^{1}\right) \cap L^{\infty}(0, T ; Y)$ to the coagulation equation (5.1), (1.8), (5.2). Moreover, the associated function "in rescaled variables" $g$, defined by (1.19), is a solution to the self-similar coagulation equation (5.5) and satisfies

$$
(g(t))_{t \geqslant 1} \text { is uniformly bounded in } X_{k} \forall k \geqslant 2-\beta .
$$

The main idea of the proof is to obtain Theorem 5.1 from Theorem 4.1 using a singular perturbation limit, more precisely using a Fokker-Planck like asymptotic of the coagulation-fragmentation model.

Proof of Theorem 5.1. Point 1. First notice that, by a simple homogeneity argument, a solution $g$ of the "evolution self-similar coagulation" equation (5.5) satisfies the a priori bounds established in Lemmas 4.2 and 4.3 for the solution of the coagulation fragmentation equation (4.1) with fragmentation rate $\gamma=-1$. Namely, for any $g_{\text {in }} \in$ $\Xi_{0} \cap \dot{L}_{k}^{1}, k>2-\beta, \Xi_{0}:=\dot{L}_{\lambda}^{1} \cap \dot{L}_{2-\beta}^{1}$, and for any $\tau \geqslant 0$, there exists $v_{\tau}:=v_{\tau}\left(\left\|g_{\text {in }}\right\|_{\Xi_{0}}, a, k, \tau\right)$ such that

$$
\sup _{t \geqslant 0}\|g(t, .)\|_{X_{k}} \leqslant \max \left(v_{0},\left\|g_{\text {in }}\right\|_{X_{k}}\right) \quad \text { and } \quad \sup _{t \geqslant \tau}\|g(t, .)\|_{X_{k}} \leqslant v_{\tau} \quad \text { for } \tau>0 .
$$

Let us introduce now the fragmentation kernel $L_{\varepsilon}$, associated to the kernel $b_{\varepsilon}$, defined by

$$
b_{\varepsilon}\left(y, y^{\prime}\right):=y^{-1} B_{\varepsilon}\left(y^{\prime} / y\right), \quad B_{\varepsilon}(z):=2 \varepsilon^{-2} \mathbf{1}_{1-\varepsilon<z<1} .
$$

A straightforward computation shows that, for all $k \in \mathbb{R}$,

$$
L_{\varepsilon}^{*} y^{k}=(1-k) y^{k}+y^{k} \mathcal{O}(\varepsilon), \quad \text { as } \varepsilon \rightarrow 0 .
$$

For any $\varepsilon>0$, let then consider $G_{\varepsilon} \in X_{\infty}$ the stationary solution to the coagulation-fragmentation equation

$$
\partial_{t} g_{\varepsilon}=C\left(g_{\varepsilon}\right)+L_{\varepsilon} g_{\varepsilon},
$$

such that $M_{1}\left(G_{\varepsilon}\right)=\rho$. The existence of $G_{\varepsilon}$ has been established in Theorem 4.1. Due to the form of (5.11) we easily see that the estimates (5.9) hold for $\left(g_{\varepsilon}\right)$, uniformly with respect to $\varepsilon>0$. That implies (one has to come back to how it has been established the existence of $G_{\varepsilon}$ in the proof of Theorem 4.1) that

$$
\left(G_{\varepsilon}\right) \text { is bounded in } X_{k} \text { for any } k \geqslant 2-\beta \text {. }
$$

By compactness, there exists a subsequence $\left(G_{\varepsilon_{k}}\right)$ and a measure $G \in X_{\infty}$ such that $M_{1}(G)=\rho$ and $G_{\varepsilon_{k}} \rightarrow G$ weakly in $X_{k}$ for any $k \geqslant 2-\beta$. On the one hand, thanks to Theorem 2.7 we may pass to the limit in the coagulation kernel and then, for any $\phi \in C_{c}^{2}$,

$$
\left\langle C\left(G_{\varepsilon_{k}}\right), \phi\right\rangle \rightarrow\langle C(G), \phi\rangle \quad \text { when } k \rightarrow \infty,
$$

where $\langle C(G), \phi\rangle$ stands for the dual formulation of the coagulation kernel which makes sense because $G \in M_{\lambda}^{1} \cap$ $M_{2-\beta}^{1}$.

On the other hand, for any $\phi \in C_{c}^{2}(0, \infty)$, we have

$$
\left\langle L_{\varepsilon} G_{\varepsilon}, \phi\right\rangle=\left\langle G_{\varepsilon}, L_{\varepsilon}^{*} \phi\right\rangle, \quad L_{\varepsilon}^{*} \phi=\int_{0}^{y} b_{\varepsilon}\left(y, y^{\prime}\right)\left(\phi^{\prime}-\frac{y^{\prime}}{y} \phi\right) d y^{\prime} .
$$

By Taylor expansion $\phi\left(y^{\prime}\right)=\phi(y)+\left(y^{\prime}-y\right) \phi_{y}(y)+\left(y^{\prime}-y\right)^{2} \phi_{y y}(\zeta) / 2$ for some $\zeta=\zeta\left(y, y^{\prime}\right)>0$, so that 


$$
\begin{aligned}
L_{\varepsilon}^{*} \phi & =\int_{0}^{y} \frac{b_{\varepsilon}\left(y, y^{\prime}\right)}{y}\left[\left(y-y^{\prime}\right) \phi+\left(y^{\prime}-y\right) y \phi_{y}(y)+y \frac{\left(y^{\prime}-y\right)^{2}}{2} \phi_{y y}(\zeta)\right] d y^{\prime} \\
& =\phi(y) S_{\varepsilon}(y)-y \phi_{y}(y) S_{\varepsilon}(y)+T_{\varepsilon}
\end{aligned}
$$

with

$$
S_{\varepsilon}(y):=\int_{0}^{y} b_{\varepsilon}\left(y, y^{\prime}\right)\left(1-y^{\prime} / y\right) d y^{\prime}=y^{\gamma+1} \int_{0}^{1} B_{\varepsilon}(\sigma)(1-\sigma) d \sigma=1
$$

and

$$
T_{\varepsilon}(y):=\int_{0}^{y} b_{\varepsilon}\left(y, y^{\prime}\right) \frac{\left(y^{\prime}-y^{\prime}\right)^{2}}{2} \phi_{y y}(\zeta) d y^{\prime} \leqslant y^{\gamma+3}\left\|\phi_{y y}\right\|_{L^{\infty}} \int_{0}^{1} B_{\varepsilon}(z)(1-z)^{2} d z=\mathcal{O}(\varepsilon) y^{\gamma+3} .
$$

As a conclusion,

$$
L_{\varepsilon}^{*} \phi \rightarrow \phi-y \phi_{y} \quad \text { uniformly in }(0, \infty)
$$

and therefore

$$
\left\langle L_{\varepsilon_{k}} G_{\varepsilon_{k}}, \phi\right\rangle=\left\langle G_{\varepsilon_{k}}, L_{\varepsilon_{k}}^{*} \phi\right\rangle \rightarrow\left\langle G, \phi-y \phi_{y}\right\rangle=\langle D G, \phi\rangle .
$$

Using (5.14) and (5.15) we can pass to the limit in the stationary equation associated to Eq. (5.12), and we obtain that $G$ is a dual solution to the self-similar profile coagulation equation (5.4).

Proof of Theorem 5.1. Point 2. First notice that, for the coagulation equation (5.1), the a priori estimates are

$$
\sup _{t \geqslant 0}\|f\|_{L^{2}} \leqslant\left\|f_{\text {in }}\right\|_{L^{2}} \quad \text { and } \quad \sup _{t \in[0, T]}\|f\|_{L_{1}^{1} \cap X_{k}} \leqslant C_{T}
$$

for a constant $C_{T}=C_{T}\left(\left\|f_{\text {in }}\right\|_{L_{k}^{1}}, a, b, k\right)$ for any $T>0$ and any $k \geqslant \lambda$. See [34,42] for the former and just copy the proof of Lemma 4.2 for the last ones. A first consequence is that for any given $f_{\text {in }} \in L^{\infty}$ with compact support included in $(0, \infty)$, one may build a solution $f \in C([0, \infty) ; Y)$ satisfying (5.16), see [36], which is unique thanks to Theorem 2.9. A second consequence is that the solution $g$ in self-similar variables associated to $f$ satisfies the local analogy to the estimates (5.16). That is enough (because of the regularity of $g$ ) to compute once again in a rigorous way the formal derivative of the estimates stated in Lemma 4.2. Therefore $g$ satisfies (5.8).

Now, for a given $f_{\text {in }} \in Y$, we approximate it by the sequence $\left(f_{\text {in }}^{\varepsilon}\right)$ defined by $f_{\text {in }}^{\varepsilon}:=\min \left(f_{\text {in }}, \varepsilon^{-1}\right) \mathbf{1}_{\left[\varepsilon, \varepsilon^{-1}\right]}$. The associated solutions $f^{\varepsilon}, g^{\varepsilon} \in C\left([0, \infty) ; L^{1}(Y)\right)$ satisfy the conclusion of Theorem 5.1 with uniform bound with respect to $\varepsilon>0$ thanks to the previous step, and they are Cauchy sequences thanks to the uniqueness Theorem 2.9. We conclude, passing to the limit $\varepsilon \rightarrow 0$.

\section{Acknowledgements}

The two first authors gratefully acknowledge the partial support of the European Research Training Network HYKE HPRN-CT-2002-00282 during this work. The first author was partially supported by the CICYT under grant BFM2002-03345. 


\section{Appendix A. From fragmentation mechanism to fragmentation operator}

The aim of this section is to present a simple derivation of the fragmentation operator (1.4) from a more intuitive and precise description of the fragmentation mechanism. Let us then consider a system of many particles which are only characterized by their sizes. We denote by $\{y\}$ any particle of size $y \in(0, \infty)$. A linear fragmentation reaction is a microscopic event in which a single particle $\{y\}$ breaks a part in a family of smaller particles in such a way that the total size is conserved. Schematically, it can be written as

$$
\{y\} \stackrel{v}{\longrightarrow}\{Y\},
$$

where $Y=\left(y_{i}\right)_{i \in \mathbb{N}^{*}}$ is one of the possible sequence of daughter fragments which stem from $\{y\}$ and satisfies

$$
y_{1} \geqslant \cdots \geqslant y_{i} \geqslant y_{i+1} \geqslant \cdots \geqslant 0 \quad \text { and } \quad y=\sum_{i=1}^{\infty} y_{i}
$$

and $v=v(y, d Y)$ is a measure on $\mathcal{S}^{\downarrow}(y)$, the set of all sequences $Y$ such that (A.2) holds. It accounts for the probability of getting a particular sequence of fragments $\{Y\}$ as the result of the fragmentation of $\{y\}$.

At the (larger) mesoscopic level, the system of particles is described by the concentration density $f(t, y) \geqslant 0$ of particles of size $y \in(0, \infty)$ at time $t$. The evolution of the system which undergoes the only fragmentation microscopic mechanism may then be written as

$$
\frac{\partial f}{\partial t}(t, y)=\mathcal{L} f(t, .)(y)
$$

where $(\mathcal{L} f)(y)$ is the infinitesimal fragmentation operator which accounts for the creation-annihilation of particles of size $y$ due to fragmentation. The fragmentation operator splits into two terms

$$
\mathcal{L} f=\mathcal{L}^{+} f-\mathcal{L}^{-} f
$$

where the gain term $\left(\mathcal{L}^{+} f\right)(y)$ accounts for all fragmentation events which give rise to a particle of size $y \in(0, \infty)$, while the loss term $\left(\mathcal{L}^{-} f\right)(y)$ counts all possible fragmentation of a particle of size $y \in(0, \infty)$. Let us introduce $v_{i}$ the $i$ th marginal measure of $v$, which is defined by

$$
\forall \phi \in C_{b}((0, \infty)) \quad \int_{0}^{\infty} \phi\left(y^{\prime}\right) v_{i}\left(y, d y^{\prime}\right)=\int_{\mathcal{S} \downarrow(y)} \phi\left(y_{i}\right) v(y ; d Y) .
$$

The meaning of $v_{i}$ is the following. For any $y, y^{\prime} \in(0, \infty), v_{i}\left(y ; d y^{\prime}\right)$ stands for the rate of creation of a daughter particle $\left\{y^{\prime}\right\}$ as the $i$ th fragment (with decreasing order) of the particle $\{y\}$ or, in other words, $y_{i}=y^{\prime}$, where $Y=\left(y_{i}\right)_{i} \geqslant 1$ is the sequence of daughter fragments defined in (A.1)-(A.2).

The infinitesimal fragmentation operator is then given by

$$
(\mathcal{L} f)(y):=\sum_{j=1}^{\infty} \int_{0}^{\infty} f^{\prime \prime} v_{j}\left(y^{\prime \prime} ; d y\right) d y^{\prime \prime}-f(y) \int_{\mathcal{S} \downarrow(y)} v(y ; d Y) .
$$

We introduce now $b$, the rate of creation of a particle $\left\{y^{\prime}\right\}$ as a fragment of the particle $\{y\}$ given by

$$
b\left(y, d y^{\prime}\right):=\sum_{j=1}^{\infty} v_{j}\left(y, d y^{\prime}\right)
$$

Notice that 


$$
\begin{aligned}
y \int_{\mathcal{S} \downarrow(y)} v(y, d Y) & =\int_{\mathcal{S} \downarrow(y)}\left(\sum_{j=1}^{\infty} y_{j}\right) v(y, d Y)=\sum_{j=1}^{\infty} \int_{\mathcal{S} \downarrow(y)} y_{j} v(y, d Y) \\
& =\sum_{j=1}^{\infty} \int_{0}^{\infty} y^{\prime} v_{j}\left(y, d y^{\prime}\right)=\int_{0}^{\infty} y^{\prime}\left(\sum_{j=1}^{\infty} v_{j}\left(y, d y^{\prime}\right)\right)=\int_{0}^{\infty} y^{\prime} b\left(y, d y^{\prime}\right),
\end{aligned}
$$

from where we obtain

$$
(\mathcal{L} f)(y)=\int_{0}^{\infty} f^{\prime \prime} b\left(y^{\prime \prime} ; d y\right) d y^{\prime \prime}-f(y) \int_{0}^{\infty} \frac{y^{\prime}}{y} b\left(y, d y^{\prime}\right) .
$$

Since we have the evident support condition $\operatorname{supp} b(y,.) \subset[0, y]$ for any $y>0$, we have thus obtained precisely the expression of $L$ given in (1.4).

On the other hand, for any test function $\phi \in C_{b}((0, \infty))$, there holds

$$
\begin{aligned}
\int_{0}^{\infty} \phi(y) \sum_{j=1}^{\infty} \int_{0}^{\infty} f^{\prime \prime} v_{j}\left(y^{\prime \prime} ; d y\right) d y^{\prime \prime} & =\sum_{j=1}^{\infty} \int_{0}^{\infty} \int_{0}^{\infty} \phi\left(y^{\prime}\right) f(y) v_{j}\left(y ; d y^{\prime}\right) d y \\
& =\sum_{j=1}^{\infty} \int_{0}^{\infty} \int_{\mathcal{S} \downarrow} \phi(y)
\end{aligned}
$$

and there also holds

$$
\int_{0}^{\infty} \phi(y) f(y) \int_{\mathcal{S} \downarrow(y)} v(y ; d Y) d y=\sum_{j=1}^{\infty} \int_{0}^{\infty} \int_{\mathcal{S} \downarrow(y)} \frac{y_{i}}{y} v(y ; d Y) \phi(y) f(y) d y .
$$

We obtain therefore the following expression for the dual formulation of the fragmentation operator

$$
\langle\mathcal{L} f, \phi\rangle=\left\langle f, \mathcal{L}^{*} \phi\right\rangle,
$$

with

$$
\mathcal{L}^{*} \phi:=\int_{\mathcal{S} \downarrow(y)} \sum_{j=1}^{\infty}\left(\phi\left(y_{i}\right)-\frac{y_{i}}{y} \phi(y)\right) v(y ; d Y) .
$$

In particular, for a self-similar fragmentation rate $v$ in the sense of [9], that is $v(y, d Y)=\tau(y) \mu(d S)$ with $S=Y / y$ and $\tau(y)=y^{\alpha}, \alpha \in \mathbb{R}$, or more precisely, such that:

$$
\forall \phi \in C_{b}\left(\mathbb{R}^{\mathbb{N}}\right) \quad \int_{\mathcal{S} \downarrow(y)} \phi(Y) v(y, d Y)=\tau(y) \int_{\mathcal{S} \downarrow(1)} \phi(y S) \mu(d S),
$$

we recover the fragmentation operator expression of $[9,29]$

$$
\mathcal{L}^{*} \phi:=\tau(y) \int_{\mathcal{S} \downarrow(1)} \sum_{j=1}^{\infty}\left(\phi\left(y s_{i}\right)-s_{j} \phi(y)\right) \mu(d S) .
$$

Our final aim is to make a link between the general formalism presented here with the (most usual) binary fragmentation mechanism. A binary fragmentation mechanism is describe by (A.1)-(A.2) where the measure $v$ satisfies

$$
\operatorname{supp} v(y, .) \subset \mathcal{S}_{2}^{\downarrow}(y)
$$


with $\mathcal{S}_{2}^{\downarrow}(y):=\left\{Y=\left(y_{k}\right)_{k \geqslant 1} \in \mathcal{S}^{\downarrow} ; \quad y_{k}=0 \forall k \geqslant 3\right\} \approx\left\{\left(y_{1}, y_{2}\right) \in \mathbb{R}_{+}, y_{1}+y_{2}=y\right\}$. For any test function $\phi \in$ $C([0, y])$, there holds

$$
\begin{aligned}
\int_{0}^{y} \nu_{1}\left(y, y^{\prime}\right) \phi^{\prime} d y^{\prime} & =\int_{\mathcal{S} \downarrow(y)} \phi\left(y_{2}\right) v(y, d Y)=\int_{\mathcal{S} \downarrow(y)} \phi\left(y_{2}\right) \mathbf{1}_{Y \in \mathcal{S}_{2}^{\downarrow}} v(y, d Y) \\
& =\int_{\mathcal{S} \downarrow(y)} \phi\left(y-y_{1}\right) v(y, d Y)=\int_{0}^{y} \phi\left(y-y^{\prime}\right) \nu_{2}\left(y, y^{\prime}\right) d y^{\prime} \\
& =\int_{0}^{y} \phi\left(y^{\prime}\right) \nu_{2}\left(y, y-y^{\prime}\right) d y^{\prime} .
\end{aligned}
$$

This implies the main fundamental symmetry property

$$
v_{1}\left(y, y^{\prime}\right)=v_{2}\left(y, y-y^{\prime}\right) \quad \forall y^{\prime} \in[0, y] .
$$

One can also show that $v$ is such that

$$
\operatorname{supp} v_{1}(y, .) \subset[y / 2, y], \quad \operatorname{supp} v_{2}(y, .) \subset[0, y / 2], \quad \text { and } \quad v_{k} \equiv 0 \quad \forall k \geqslant 3 .
$$

Let define, as before,

$$
b\left(y, y^{\prime}\right):=\sum_{j=1}^{\infty} v_{j}\left(y, y^{\prime}\right)=v_{1}\left(y, y^{\prime}\right)+v_{2}\left(y, y^{\prime}\right) .
$$

We observe that, thanks to (A.5) $b$ enjoys the symmetry property

$$
b\left(y, y-y^{\prime}\right)=v_{1}\left(y, y-y^{\prime}\right)+v_{2}\left(y, y-y^{\prime}\right)=v_{2}\left(y, y^{\prime}\right)+v_{1}\left(y, y^{\prime}\right)=b\left(y, y^{\prime}\right) .
$$

We finally introduce

$$
\beta\left(y, y^{\prime}\right)=b\left(y+y^{\prime}, y\right),
$$

the rate of formation of a pair of particles of size $\left(y^{\prime}, y\right)$ as the breakage result of a particle of size $y+y^{\prime}$. We deduce from (A.6) that $\beta$ enjoys the symmetry property

$$
\beta\left(y, y^{\prime}\right)=\beta\left(y^{\prime}, y\right) \text {. }
$$

Putting $\beta$ in (A.4), we find

$$
(\mathcal{L} f)(y)=\int_{y}^{\infty} f^{\prime \prime} \beta\left(y, y^{\prime \prime}-y\right) d y^{\prime \prime}-f(y) \int_{0}^{y} \frac{y^{\prime}}{y} \beta\left(y^{\prime}, y-y^{\prime}\right) d y^{\prime} .
$$

Making the change of variables $y^{\prime} \rightarrow y-y^{\prime}$ and using the symmetry property (A.7), we observe that

$$
\int_{0}^{y} \frac{y^{\prime}}{y} \beta\left(y^{\prime}, y-y^{\prime}\right) d y^{\prime}=\int_{0}^{y} \frac{y-y^{\prime}}{y} \beta\left(y-y^{\prime}, y^{\prime}\right) d y^{\prime},
$$

from which we deduce

$$
\int_{0}^{y} \frac{y^{\prime}}{y} \beta\left(y^{\prime}, y-y^{\prime}\right) d y^{\prime}=\frac{1}{2} \int_{0}^{y} \beta\left(y-y^{\prime}, y^{\prime}\right) d y^{\prime} .
$$


Gathering (A.8) and (A.9), we then recover the usual expression for the binary fragmentation operator, namely

$$
(\mathcal{L} f)(y)=\int_{y}^{\infty} f^{\prime \prime} \beta\left(y, y^{\prime \prime}-y\right) d y^{\prime \prime}-\frac{1}{2} \int_{0}^{y} \beta\left(y^{\prime}, y-y^{\prime}\right) d y^{\prime} f(y) .
$$

\section{References}

[1] D.J. Aldous, Deterministic and stochastic models for coalescence (aggregation, coagulation): a review of the mean-field theory for probabilists, Bernoulli 5 (1999) 3-48.

[2] H. Amann, Ordinary Differential Equations. An Introduction to Nonlinear Analysis, Translated from the German by Gerhard Metzen, de Gruyter Studies in Mathematics, vol. 13, Walter de Gruyter, Berlin, 1990.

[3] L. Arlotti, J. Banasiak, Strictly substochastic semigroups with application to conservative and shattering solutions to fragmentation equations with mass loss, J. Math. Anal. Appl. 293 (2) (2004) 693-720.

[4] M. Balabane, Systèmes différentiels, cours de l'Ecole Nationale des Ponts et Chaussées, 1982.

[5] N. Ben Abdallah, M. Escobedo, S. Mischler, Convergence to the equilibrium for the Pauli equation without detailed balance condition, in preparation.

[6] J. Bertoin, On small masses in self-similar fragmentations, Stochastic Process. Appl. 109 (1) (2004) 13-22.

[7] J. Bertoin, The asymptotic behavior of fragmentation processes, J. Eur. Math. Soc. (JEMS) 5 (4) (2003) 395-416.

[8] J. Bertoin, Eternal solutions to Smoluchowski's coagulation equation with additive kernel and their probabilistic interpretations, Ann. Appl. Probab. 12 (2) (2002) 547-564.

[9] J. Bertoin, Self-similar fragmentations, Ann. Inst. H. Poincaré Probab. Statist. 38 (3) (2002) 319-340.

[10] J. Bertoin, Homogeneous fragmentation processes, Probab. Theory Related Fields 121 (3) (2001) 301-318.

[11] T. Cazenave, A. Haraux, An Introduction to Semilinear Evolution Equations, Oxford Lecture Series in Mathematics and its Applications, vol. 13, The Clarendon Press, Oxford University Press, New York, 1998.

[12] F.P. da Costa, On the dynamic scaling behavior of solutions to the discrete Smoluchowski equations, Proc. Edinburgh Math. Soc. 39 (2) (1996) 547-559.

[13] M. Deaconu, E. Tanré, Smoluchowski's coagulation equation: probabilistic interpretation of solutions for constant, additive and multiplicative kernels, Ann. Scuola Norm. Sup. Pisa Cl. Sci. 29 (4) (2000) 549-579.

[14] B. Derrida, C. Godréche, I. Yekuitieli, Scale-invariant regimes in one-dimensional models of growing and coalescing droplets, Phys. Rev. A 44 (1991) 6241-6251.

[15] R.J. DiPerna, P.-L. Lions, Ordinary differential equations, transport theory and Sobolev spaces, Invent. Math. 98 (1989) $707-741$.

[16] P.G.J. van Dongen, M.H. Ernst, Cluster size distribution in irreversible aggregation at large times, J. Phys. A 18 (1985) $2779-2793$.

[17] P.G.J. van Dongen, M.H. Ernst, Scaling solutions of Smoluchowski's coagulation equation, J. Statist. Phys. 50 (1988) $295-329$.

[18] R.L. Drake, A general mathematical survey of the coagulation equation, in: Topics in Current Aerosol Research (part 2), International Reviews in Aerosol Physics and Chemistry, Pergamon Press, Oxford, 1972, pp. 203-376.

[19] P.B. Dubovskiŭ, I.W. Stewart, Trend to equilibrium for the coagulation-fragmentation equation, Math. Methods Appl. Sci. 19 (1996) $761-772$.

[20] R.E. Edwards, Functional Analysis, Theory and Applications, Holt, Rinehart and Winston, 1965.

[21] A. Eibeck, W. Wagner, Stochastic particle approximations for Smoluchowski's coagulation equation, Ann. Appl. Probab. 11 (2001) $1137-$ 1165 .

[22] M. Escobedo, Ph. Laurençot, S. Mischler, B. Perthame, Gelation and mass conservation in coagulation-fragmentation models, J. Differential Equations 195 (1) (2003) 143-174.

[23] M. Escobedo, S. Mischler, B. Perthame, Gelation in coagulation and fragmentation models, Comm. Math. Phys. 231 (2002) 157-188.

[24] M. Escobedo, E. Zuazua, Large time behavior of the solutions of a convection diffusion equation, J. Funct. Anal. 100 (1991) 119-161.

[25] N. Fournier, J.-S. Giet, On small particles in coagulation-fragmentation equations, J. Statist. Phys. 111 (5-6) (2003) 1299-1329.

[26] N. Fournier, S. Mischler, Trend to the equilibrium for the coagulation equation with strong fragmentation but with balance condition, in: Proceedings: Mathematical, Physical and Engineering Sciences, in press.

[27] N. Fournier, S. Mischler, On a Boltzmann equation for elastic, inelastic and coalescing collisions, preprint, 2003, submitted for publication.

[28] I.M. Gamba, V. Panferov, C. Villani, On the Boltzmann equation for diffusively excited granular media, Comm. Math. Phys. 246 (3) (2004) 503-541.

[29] B. Haas, Loss of mass in deterministic and random fragmentations, Stochastic Process. Appl. 106 (2) (2003) $245-277$.

[30] M. Kreer, O. Penrose, Proof of dynamical scaling in Smoluchowski's coagulation equation with constant kernel, J. Statist. Phys. 75 (1994) 389-407.

[31] D.S. Krivitsky, Numerical solution of the Smoluchowski kinetic equation and asymptotics of the distribution function, J. Phys. A 28 (1995) 2025-2039. 
[32] $\mathrm{Ph}$. Laurençot, Convergence to self-similar solutions for coagulation equation, preprint, 2003.

[33] Ph. Laurençot, S. Mischler, From the discrete to the continuous coagulation-fragmentation equations, Proc. Roy. Soc. Edinburgh Sect. A 132 (5) (2002) 1219-1248.

[34] Ph. Laurençot, S. Mischler, The continuous coagulation-fragmentation equations with diffusion, Arch. Rational Mech. Anal. 162 (2002) 45-99.

[35] Ph. Laurençot, S. Mischler, Convergence to equilibrium for the continuous coagulation-fragmentation equation, Bull. Sci. Math. 127 (2003) 179-190.

[36] P. Laurençot, S. Mischler, On coalescence equations and related models, in: P. Degond, L. Pareschi, G. Russo (Eds.), Modelling and Computational Methods for Kinetic Equations, in: Series Modelling and Simulation in Science, Engineering and Technology (MSSET), Birkhäuser, 2004, submitted for publication.

[37] F. Leyvraz, Existence and properties of post-gel solutions for the kinetic equations of coagulation, J. Phys. A 16 (1983) $2861-2873$.

[38] F. Leyvraz, Scaling theory and exactly solved models in the kinetics of irreversible aggregation, Phys. Reports 383 (2-3) (2003) 95-212.

[39] G. Menon, R.L. Pego, Approach to self-similarity in Smoluchowski's coagulation equation, preprint, 2003.

[40] G. Menon, R.L. Pego, Dynamical scaling in Smoluchowski's coagulation equation: uniform convergence, preprint, 2003.

[41] S. Mischler, C. Mouhot, M. Rodriguez Ricard, Cooling process for inelastic Boltzmann equations, in preparation.

[42] S. Mischler, M. Rodriguez Ricard, Existence globale pour l'équation de Smoluchowski continue non homogène et comportement asymptotique des solutions, C. R. Acad. Sci. Paris Sér. I Math. 336 (2003) 407-412.

[43] S. Mischler, B. Wennberg, On the spatially homogeneous Boltzmann equation, Ann. Inst. H. Poincaré Anal. Non Linéaire 16 (4) (1999) 467-501.

[44] H. Tanaka, S. Inaba, K. Nakaza, Steady-state size distribution for self-similar collision cascade, Icarus 123 (1996) 450-455. 